\title{
Measuring the Liquidity of ETFs: An Application to the European Market
}

\author{
Thierry Roncalli \\ Quantitative Research \\ Lyxor Asset Management, Paris \\ thierry.roncalli@lyxor.com \\ Ban Zheng \\ Quantitative Research \\ Lyxor Asset Management, Paris \\ ban.zheng@lyxor.com \\ February 2014
}

\begin{abstract}
The liquidity of exchange traded funds is of utmost importance for regulators, investors and providers. However, the study of liquidity is still in its infancy. In this work, we show some stylised facts of liquidity statistics (daily/intraday spread, trading volume, etc.). We also propose a new liquidity measure combining these statistics. In this case, liquidity is a power function of the spread where the parameters are determined by actual trading volumes. We also study the relationship between the liquidity of ETFs and the liquidity of the underlying index. We show that they are correlated on a daily basis, but not in terms of intraday frequency. We also define a measure of liquidity improvement and apply it to the EURO STOXX 50 index.
\end{abstract}

Keywords: Exchange traded fund, liquidity, spread, trading volume, order book, liquidity improvement.

JEL classification: G11, G14.

\section{Introduction}

Exchange traded funds (ETFs) are mutual funds that are traded on an exchange. They generally replicate the performance of an index ${ }^{1}$. They allow investors to gain exposure to several asset classes on a real-time basis, meaning that shares in an ETF can be bought and sold through a broker-dealer in the same way as stocks. This ability to provide intraday exposure explains the incredible growth of the ETF market in the last ten years. According to ETFGI (2014), there were 3, 594 ETFs worldwide with assets of US $\$ 2,254$ billion at the end of $2013^{2}$, whereas equity exposures represented about $80 \%$ of assets.

The liquidity of ETFs, which is one of the main advantages compared to index mutual funds, is however an unclear notion. Indeed, little is known about it and academic studies are very scarce on this subject. While liquidity has been extensively investigated in the stock market $^{3}$, there has been little research into the ETF market. To our knowledge, Calamia et al. (2013) is the most comprehensive study on the liquidity of ETFs. For instance, they

\footnotetext{
${ }^{1}$ According to ETFGI (2014), index-tracking ETFs represent a large part of this industry whereas active ETFs represent less than $1 \%$ of the assets in Europe.

${ }^{2}$ In this paper, we use the symbols $\mathrm{M}$ and $\mathrm{B}$ to designate respectively one million and one billion.

${ }^{3}$ See e.g. Amihud and Mendelson (1986, 1989), Brennan and Subrahmanyam (1996), Eleswarapu (1997), Amihud (2002), Sarr and Lybek (2002) and Ben-Rephael et al. (2013).
} 
show that ETF liquidity depends on index liquidity, the assets under management and the market fragmentation. Nevertheless, they use closing prices, which are less relevant than intraday prices. The aim of our paper is to understand ETF liquidity on an intraday basis. Our first task is to define the appropriate measure and its impact. In a second stage, we seek to gain a clearer understanding of the dynamics of intraday liquidity and its relationship with the liquidity of the underlying index.

The liquidity issue relating to ETFs is particular relevant in Europe. Following the rapid growth of the ETF market, it is of prime importance that investors and regulators understand its liquidity. From an investor's point of view, the goal is to evaluate the efficiency of this investment format in terms of allocation and hedging operations. Moreover, we notice today that some indexations also cover a broad universe of small, exotic and rarely traded assets. From a regulatory standpoint, the goal is to understand the impact of these instruments on the stability of the financial market. This question is particularly relevant as the proportion of market value accounted for by ETFs is growing rapidly.

In this study, we consider the universe of the 10 largest ETF providers in Europe. For each provider, we assign a number between 1 and 10 in order to obtain anonymous results. The study period is 2012. The paper is organised as follows: in section two, we provide a short presentation of limit order books in ETFs; we then estimate different liquidity measures in section three and show how spread and trading volume are related; section four is dedicated to the relationship between the liquidity of ETFs and the liquidity of the underlying assets; lastly, section five contains our conclusion.

\section{Understanding the limit order book}

ETFs are listed on an electronic system. For example, in NYSE EURONEXT, during continuous trading from 9:00 to 17:30, market orders are matched against the best limit orders on the opposite side. Various order types are accepted in electronic trading systems such as limit orders ${ }^{4}$, market orders ${ }^{5}$, stop orders ${ }^{6}$ and iceberg orders ${ }^{7}$ (Easley and O'Hara, 1991; Parlour and Seppi, 2008). Limit orders are posted to the electronic trading system and they are placed in the book according to their prices. A market order is an order that is executed immediately at the best available price in the limit order book.

The lowest price of sell orders is called the best ask, while the highest price of buy orders is called the best bid. The gap between the best ask and the best bid is called the spread. When a market order arrives, a trade occurs and the limit order book is updated. In particular, when the volume of the market order is larger than the quantity available at the corresponding best limit price, the best limit price will be modified. However, limit orders can be cancelled if they have not been executed, so the limit order book can be modified due to limit order cancellation, limit order arrival or market order arrival.

In the case of iceberg orders, the visible portion has the same priority as a regular limit order, while the hidden portion has lower priority. The hidden portion will become visible as soon as the previous visible portion is executed. It is rare that the hidden portion is consumed by a market order without previously being visible. In this study, we ignore stop

\footnotetext{
${ }^{4} \mathrm{An}$ order to be traded at a fixed price in a certain amount.

${ }^{5} \mathrm{An}$ order executed without price constraints.

${ }^{6} \mathrm{~A}$ limit or market order when a trigger price is reached.

${ }^{7}$ Only a portion of the order is visible in the order book.
} 
orders and iceberg orders, as these are relatively rare compared to limit and market order events.

It is usually the case that only the orders on the five best buy/sell limit prices are publicly available. Let $t>0$. We denote $P_{t}^{\mathrm{BID}, i}$ (or $P_{t}^{\mathrm{ASK}, i}$ ) as the $i^{\text {th }}$ bid (or ask) limit price at time $t$ and $Q_{t}^{\mathrm{BID}, i}$ (or $Q_{t}^{\mathrm{ASK}, i}$ ) as the quantity of the $i^{\text {th }}$ bid (or ask limit price) at time $t$ for $i \in\{1, \ldots, 5\}$. The mid quote $P_{t}^{\mathrm{MID}}$ at time $t$ is defined as follows:

$$
P_{t}^{\mathrm{MID}}=\frac{P_{t}^{\mathrm{BID}, 1}+P_{t}^{\mathrm{ASK}, 1}}{2}
$$

Since the data are recorded every time there is an order book event, we de note the event's mark by $M_{t} \in\{-1,0,+1\}$ where -1 represents a sell market order event, +1 represents a buy market order event and 0 represents a limit order event. The volume and price of a trade at time $t$ are respectively denoted by $Q_{t}$ and $P_{t}$. By construction, $Q_{t}$ is equal to zero when there is no trade at time $t$.

In this paper, we use a high frequency database from January 2012 to December 2012, with changes in the limit order book recorded at the microsecond level. In Figure 1, we provide a snapshot of the limit order book of the Lyxor EURO STOXX 50 ETF recorded at NYSE Euronext Paris ${ }^{8}$. It appears that the best bid is 26.325 whereas the best ask is 26.340 , meaning that the spread is 0.015 . In this case, the limit orders are not matched and there is no transaction $\left(M_{t}=0\right)$.

Figure 1: An example of a limit order book

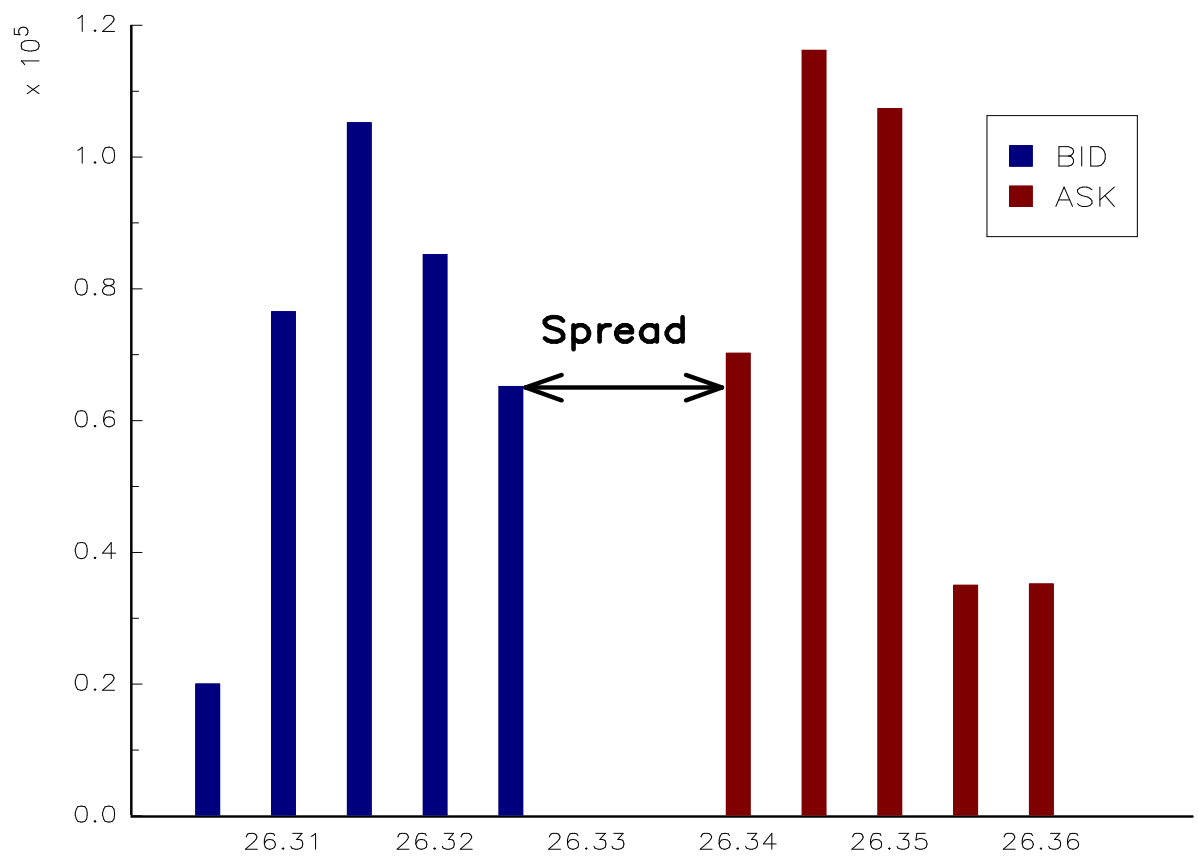

${ }^{8}$ The corresponding date is 14:00:00 and 56, 566 micro seconds on 28 December 2012. See data in Table 16 on page 30 . 
Measuring the Liquidity of ETFs: An Application to the European Market

\section{Measuring the absolute liquidity of ETFs}

Liquidity is a long-standing issue and also an elusive concept. It cannot be observed directly, because it measures the asset's ability to be sold as soon as possible without causing a significant price movement, which corresponds to the discount granted by the seller or the premium paid by the buyer ${ }^{9}$. Moreover, it is difficult to capture liquidity in a single measure. For instance, Amihud and Mendelson (1991) separate liquidity costs into four components: bid-ask spread, market impact costs, delay and search costs and direct transaction costs.

From a statistical point of view, bid-ask spread and price impact are the two measures used most frequently (Brennan and Subrahmanyam, 1996). In a limit order book, the price impact is correlated to the bid-ask spread, whereas larger excess demand has a greater impact on the market (Keim and Madhavan, 1996). Easley and O'Hara (1987) observe a similar pattern of informed traders in stock markets. Barclay et al. (1999) propose an advanced measure called CRT (cost of round-trip) which takes into account the bid-ask spread as well as the depth of the market. Inspired by these analyses of stock markets, Hassine and Roncalli (2013) compute a liquidity spread for the ETF market. Another related concept is the liquidity notional defined by Degryse et al. (2011). Below, we study these different concepts and show how they are connected.

\subsection{Volume-based liquidity measurement}

A first approach to investigate the liquidity of an ETF is to consider its volume. For instance, some investors use the assets under management (AUM) as a liquidity proxy. Contrary to the underlying idea that there is a high correlation between AUM and liquidity, AUM is not a good proxy especially in Europe, because it is an institutional market. Some ETFs may also have a large volume of assets that are predominantly held by a small number of long-term investors, which use them in their strategic asset allocation. A better solution is to consider the trading volume.

\subsubsection{Trading volume}

The trading volume is equal to $Q_{t}$ at time $t$. We define the trading volume for a given period $\left[t_{\text {begin }}, t_{\text {end }}\right]$ as follows:

$$
V=\sum_{t_{j}=t_{\mathrm{begin}}}^{t_{\text {end }}} Q_{t_{j}} P_{t_{j}}
$$

Figure 2 shows the changes of the daily volume ${ }^{10}$ for two EURO STOXX 50 ETFs. We also indicate the boxplot ${ }^{11}$ for the different ETFs. For instance, the daily volume of ETFs \#3 and \#4 is $20 \mathrm{M} €$ on average, whereas it is less than $5 \mathrm{M} €$ for ETFs \#1 and \#2. The last panel corresponds to the Lorenz curve of the trading volume when we consider the 10 largest ETFs on the EURO STOXX 50 index. The minimum (or median and maximum) curve corresponds to the daily Lorenz curve, which has the lowest (or median and highest) Gini index. These results show that the trading activity is concentrated in a very small number of ETFs. Another interesting fact is the common dynamics of the trading volumes

\footnotetext{
${ }^{9}$ This may be explained by adverse selection and inventory costs (Amihud and Mendelson, 1980; Glosten and Milgrom, 1985).

${ }^{10}$ In this work, we express trading volume in $\mathrm{M} €$.

${ }^{11}$ In this article, we adopt the following convention to represent a boxplot: the bottom and top of the box correspond to the first and third quartiles, the band inside the box is the median, while the ends of the whiskers indicate the $5^{\text {th }}$ and $95^{\text {th }}$ percentiles of the data.
} 
among the different ETF providers. The average correlation between the daily variations of the trading volume for the 10 largest EURO STOXX 50 ETFs is equal to $16.56 \%$. If we consider the four largest EURO STOXX 50 ETFs, we obtain the following correlation matrix:

\begin{tabular}{|r|rrrr|}
\hline & \multicolumn{1}{|c}{$\# 1$} & \multicolumn{1}{c}{$\# 2$} & \multicolumn{1}{c}{$\# 3$} & \multicolumn{1}{c}{$\# 4$} \\
\hline$\# 1$ & 100.00 & 16.03 & 22.66 & 27.86 \\
$\# 2$ & 16.03 & 100.00 & 31.03 & 20.59 \\
$\# 3$ & 22.66 & 31.03 & 100.00 & 39.86 \\
$\# 4$ & 27.86 & 20.59 & 39.86 & 100.00 \\
\hline
\end{tabular}

Even in this case, the correlation is very low, which indicates that trading activity on ETFs is driven more by specific factors than by common factors.

Figure 2: Trading volume statistics (EURO STOXX 50)
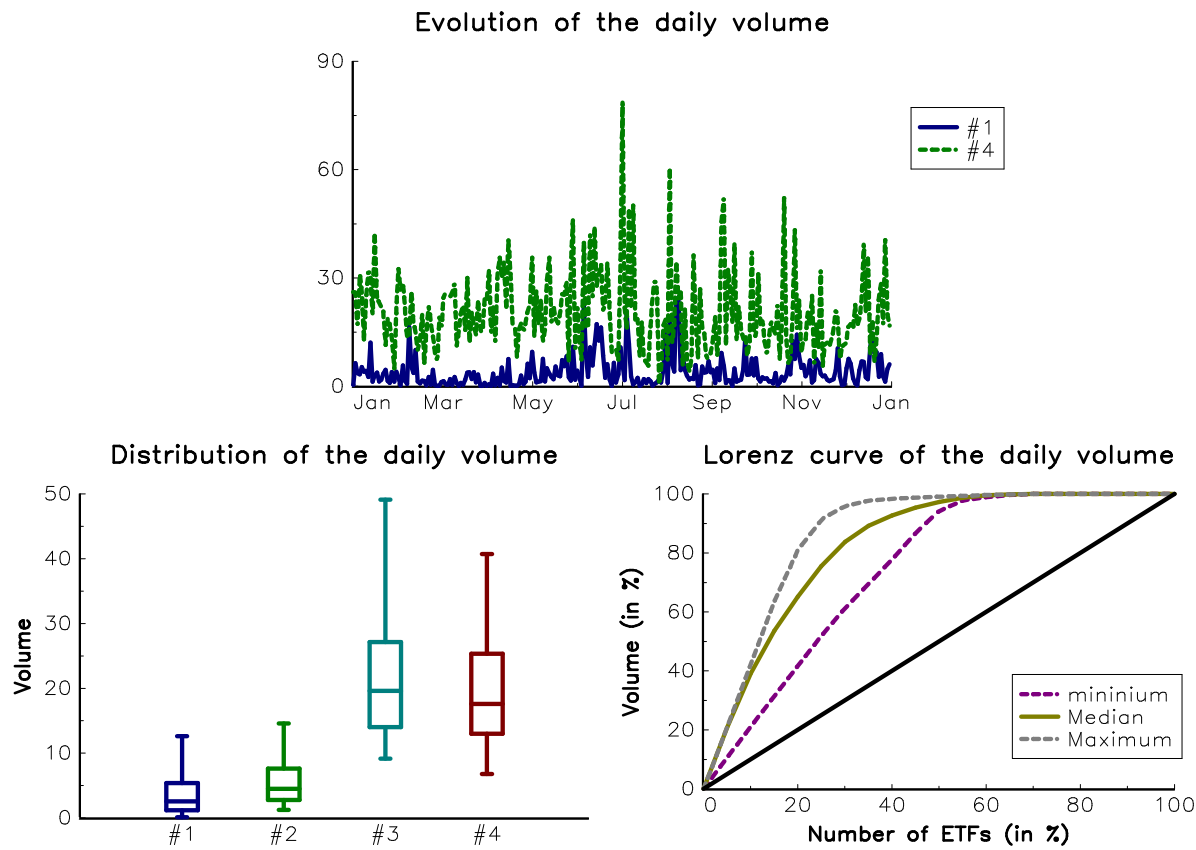

In Figure 3, we provide the intraday pattern for the trading volumes of a number of EURO STOXX 50 ETFs. For each ETF, we report the aggregated trading volume for a time period of 30 minutes. We obtain the following results:

1. Contrary to the common belief, trading activity is not concentrated at the market close and is spread throughout the day. This means that ETFs are not used as a pure substitute for index funds.

2. On the equity side, we observe that the trading volume is more significant at the market opening and close (see also Figures 21 and 22 in Appendix C on page 31). This is why we may observe a $U$ shape. We do not find this stylised fact for fixed income ETFs (see Figure 23 in Appendix C on page 32) or for markets without a daily close. 
Figure 3: Average intraday trading volume (EURO STOXX 50)

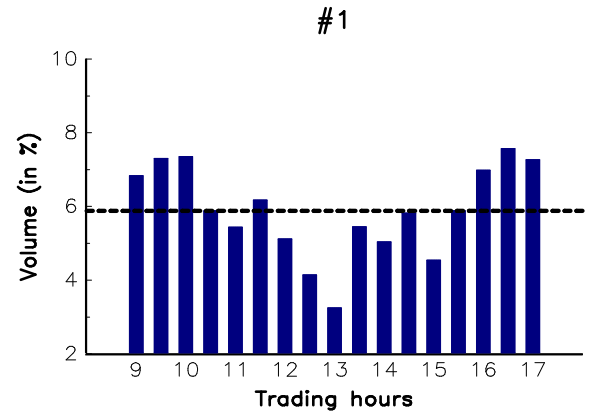

\#3

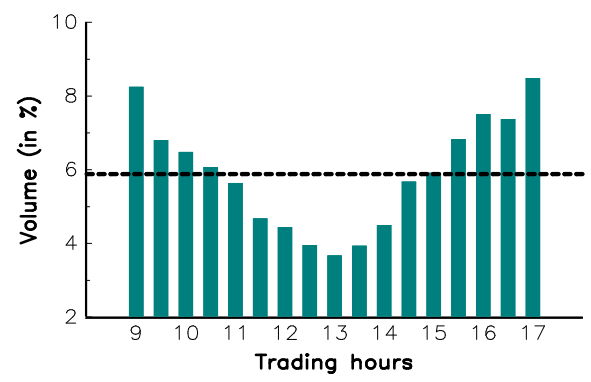

\#2

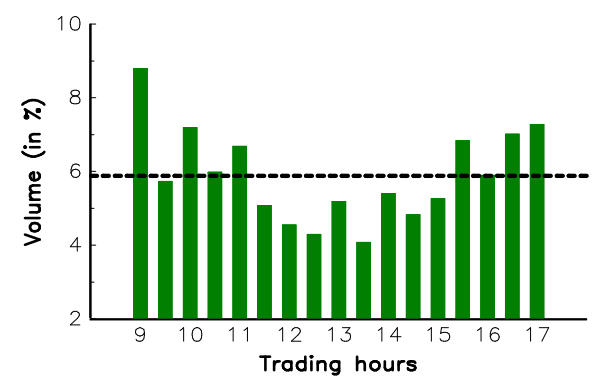

$\# 4$

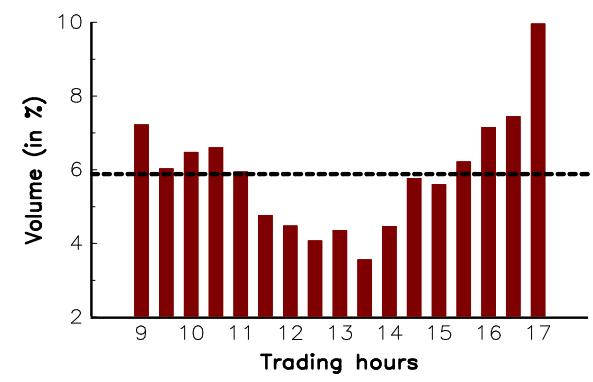

\subsubsection{Order book volume}

The volume on the best bid and ask at time $t$ is denoted by $Q_{t}^{\mathrm{BID}, 1}$ and $Q_{t}^{\mathrm{ASK}, 1}$. Let $V_{t}^{1}$ be the average volume of the best limit. We have:

$$
V_{t}^{1}=\left(\frac{Q_{t}^{\mathrm{BID}, 1}+Q_{t}^{\mathrm{ASK}, 1}}{2}\right) P_{t}^{\mathrm{MID}, 1}
$$

The volume of the best limit orders for a given period $\left[t_{\text {begin }}, t_{\text {end }}\right]$ thus corresponds to the intraday volume of the best limit orders weighted by the duration between two ticks:

$$
V^{1}=\frac{\sum_{t_{j}=t_{\mathrm{begin}}}^{t_{\text {end }}} V_{t_{j}}^{1}\left(t_{j+1}-t_{j}\right)}{\sum_{t_{j}=t_{\mathrm{begin}}}^{t_{\mathrm{end}}}\left(t_{j+1}-t_{j}\right)}
$$

In Figure 4, we report the distribution of $V^{1}$ when the reference period [ $t_{\text {begin }}, t_{\text {end }}$ ] corresponds to a trading day ${ }^{12}$. Curiously, the difference between the means is very small. On average, $V^{1}$ is equal to $2 \mathrm{M} €$ for the four largest EURO STOXX 50 ETFs. These results are surprising because they are not consistent with those obtained using trading volumes. Indeed, we might have thought that there would be a strong correlation between the trading volume and the best limit volume. Another interesting stylised fact concerns the volume disequilibrium $\mathcal{D}^{1}$ defined by the difference between the best ask and the best bid volumes:

$$
\mathcal{D}_{t}^{1}=\left(Q_{t}^{\mathrm{ASK}, 1}-Q_{t}^{\mathrm{BID}, 1}\right) P_{t}^{\mathrm{MID}, 1}
$$

\footnotetext{
${ }^{12}$ All statistics are expressed in $\mathrm{M} €$.
} 
Figure 4: Histogram of the daily best limit volume $V^{1}$ (EURO STOXX 50)
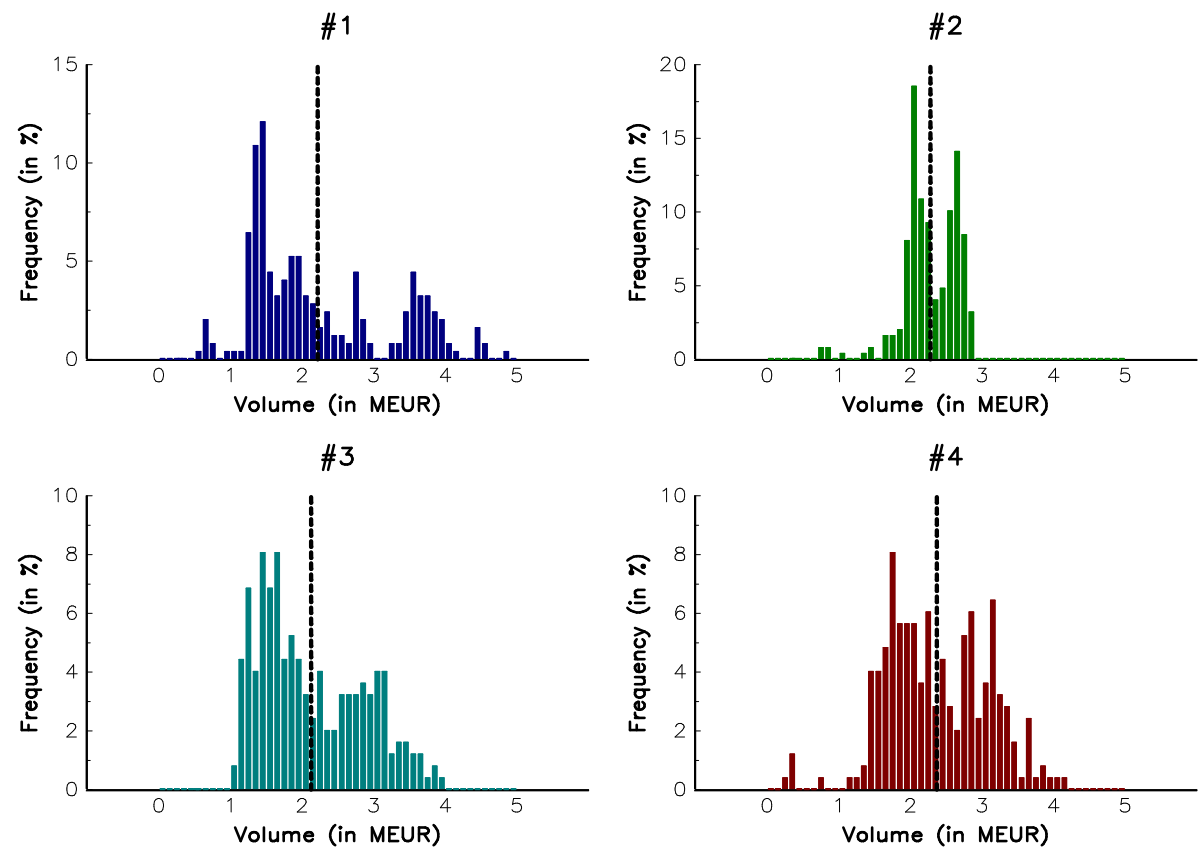

Figure 5: Scatterplot between the daily volume disequilibrium $\mathcal{D}^{1}$ (EURO STOXX 50)
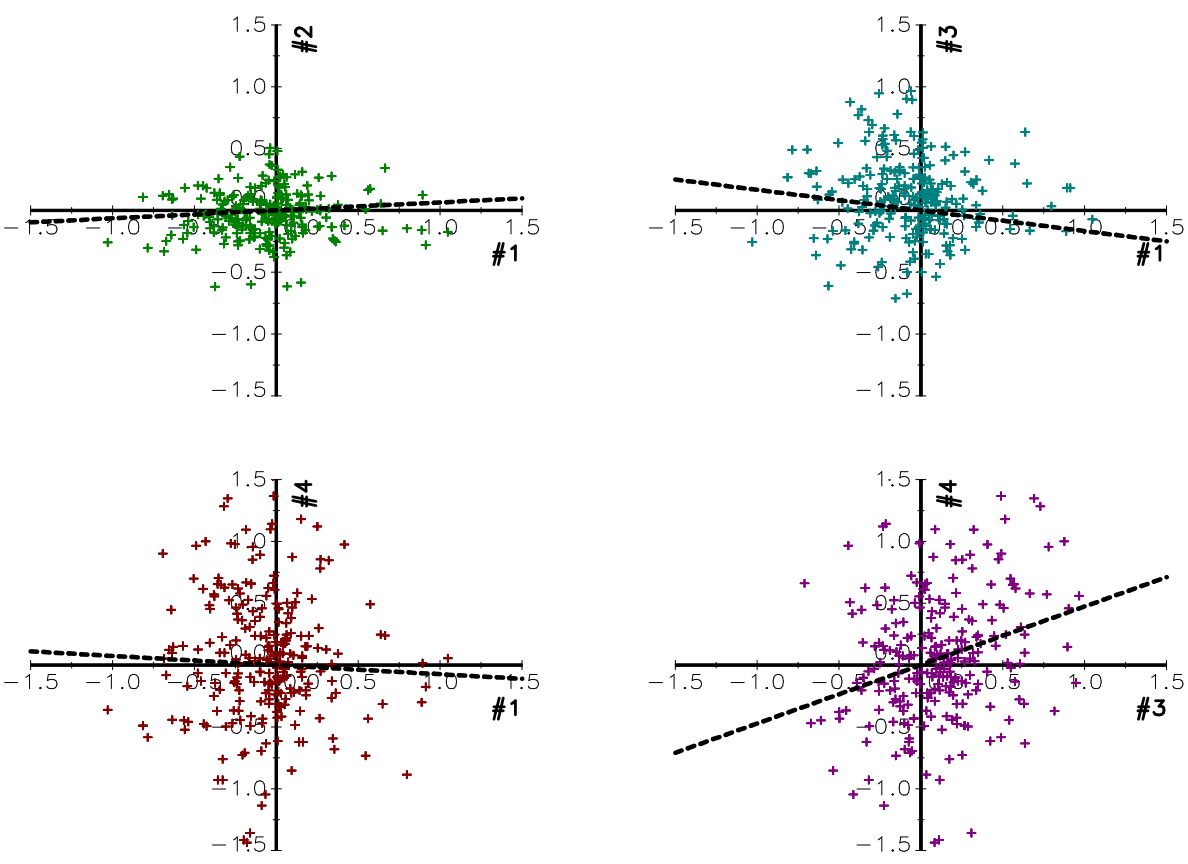
$\mathcal{D}^{1}$ reflects the difference between demand and supply. Figure 5 is the scatterplot between daily volume disequilibria. We observe that they are not quite correlated except between ETFs \#3 and \#4, which are the two largest ETFs in terms of trading volume. We may also deduce that best limit orders are a poor indicator for measuring liquidity, because they are not informative about the trading volume.

Remark 1 In Figure 4, we observe some dispersion between the best limit volumes across time. On an intraday basis, this dispersion is low meaning that these best limit volumes are not concentrated during certain specific trading hours (see Figure 24 on page 32).

\subsection{Order book-based liquidity measurement}

In this section, we consider liquidity measures based on the entire order book, rather than just on the best limit orders, because we have seen previously that the information contained in the latter is insufficient to assess the liquidity.

\subsubsection{Computing the liquidity spread}

Definition We consider the definition of the liquidity spread proposed by Hassine and Roncalli (2013). We recall that the limit order book at time $t$ is defined by the set of bid-ask prices and quantities $\left(P_{t}^{\mathrm{BID}, i}, Q_{t}^{\mathrm{BID}, i}, P_{t}^{\mathrm{ASK}, i}, Q_{t}^{\mathrm{ASK}, i}\right)$ where $i$ represents the $i^{t h}$ limit order. We denote as $N$ the notional amount that an investor would like to execute at time $t$. We define as $S_{t}(N)$ the spread at time $t$ weighted by the depth of the market as follows:

$$
S_{t}(N)=c_{t}(N) \frac{\left(\bar{P}_{t}^{\mathrm{ASK}}-\bar{P}_{t}^{\mathrm{BID}}\right)}{P_{t}^{\mathrm{MID}}}
$$

where $P_{t}^{\mathrm{MID}}$ is the mid price defined in Equation (1). The bid and ask prices $\bar{P}_{t}^{\mathrm{BID}}$ and $\bar{P}_{t}^{\mathrm{ASK}}$ correspond to:

$$
\bar{P}_{t}^{\bullet}=\frac{\sum_{i=1}^{5} \bar{Q}_{t}^{\bullet, i} P_{t}^{\bullet, i}}{\sum_{i=1}^{5} \bar{Q}_{t}^{\bullet, i}}
$$

whereas the quantities $\bar{Q}_{t}^{\mathrm{BID}, i}$ and $\bar{Q}_{t}^{\mathrm{ASK}, i}$ are given by the following relationship:

$$
\bar{Q}_{t}^{\bullet, i}=\max \left(0, \min \left(Q_{t}^{\bullet, i}, Q_{t}^{\star}-\sum_{k=1}^{i-1} Q_{t}^{\bullet, k}\right)\right)
$$

$Q_{t}^{\star}=N / P_{t}^{\mathrm{MID}}$ is the reference quantity to execute in order to target the notional $N$. The factor $c_{t}(N)$ is used to treat the case when the trading volume on the order book is lower than the notional $N$. We have:

$$
c_{t}(N)=\max \left(1, \frac{Q_{t}^{\star}}{\min \left(\sum_{i=1}^{5} Q_{t}^{\mathrm{ASK}, i}, \sum_{i=1}^{5} Q_{t}^{\mathrm{BID}, i}\right)}\right)
$$

The daily spread $S(N)$ corresponds to intraday spreads weighted by the duration between two ticks:

$$
S(N)=\frac{\sum_{t_{j}=\text { open }}^{\text {close }} S_{t_{j}}(N)\left(t_{j+1}-t_{j}\right)}{\sum_{t_{j}=\text { open }}^{\text {close }}\left(t_{j+1}-t_{j}\right)}
$$

where $S_{t_{j}}(N)$ is the spread of the $j^{\text {th }}$ tick in order to trade the notional $N$ and $\left(t_{j+1}-t_{j}\right)$ is the elapsed time between two consecutive ticks. 
Remark 2 The above framework is slightly different from the definition proposed by Hassine and Roncalli (2013). Indeed, these authors compute the mid price using the prices $\bar{P}_{t}^{\mathrm{BID}}$ and $\bar{P}_{t}^{\mathrm{ASK}}$. In this case, the mid price is endogenous. We prefer to consider the mid price by using only the best limit order.

Figure 6: Evolution of the liquidity spread with respect to the notional
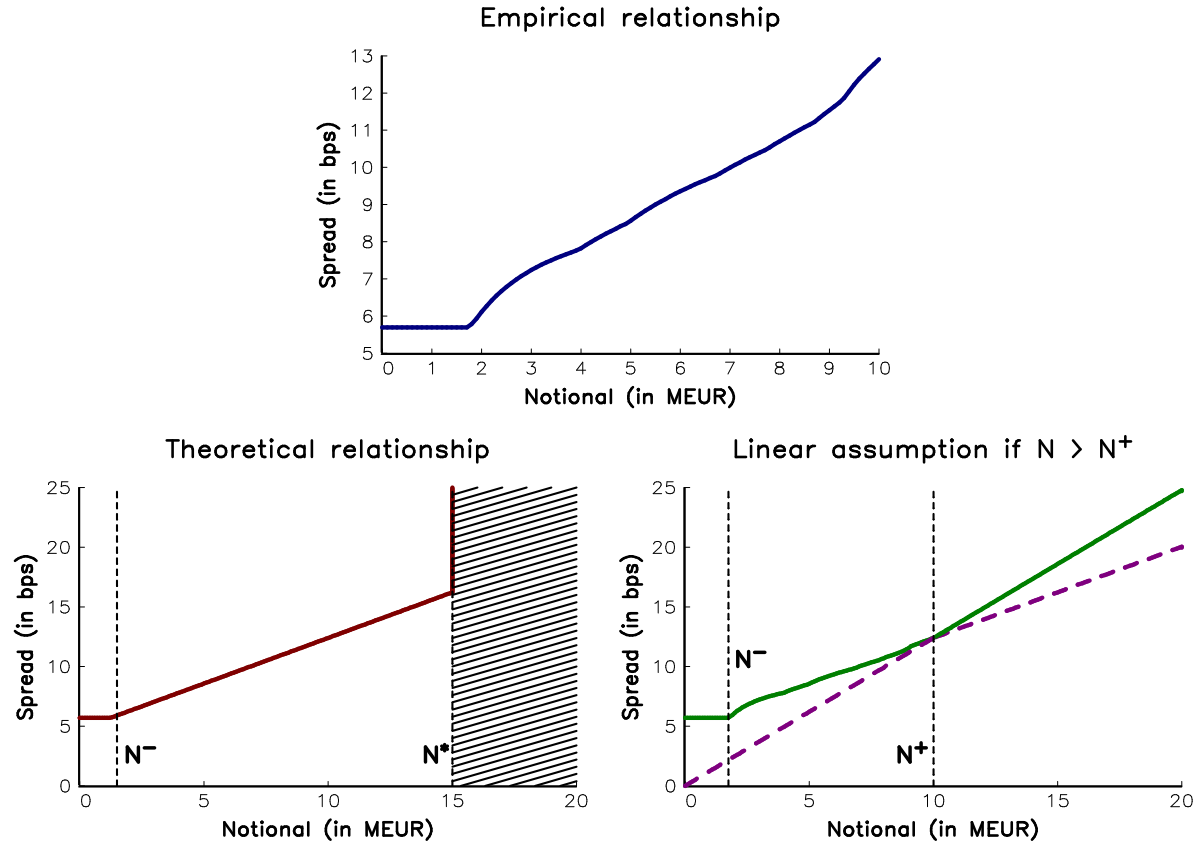

In Appendix B, we illustrate the computation of the spread by considering the limit order book used in Figure 1. In the first panel in Figure 6, we also report the evolution of the spread with respect to the given notional. We deduce the following proposition:

Proposition 1 Let us define $N^{-}$and $N^{+}$as follows:

$$
\begin{aligned}
& N^{-}=P_{t}^{\mathrm{MID}} \cdot \min \left(Q_{t}^{\mathrm{ASK}, 1}, Q_{t}^{\mathrm{BID}, 1}\right) \\
& N^{+}=P_{t}^{\mathrm{MID}} \cdot \min \left(\sum_{i=1}^{5} Q_{t}^{\mathrm{ASK}, i}, \sum_{i=1}^{5} Q_{t}^{\mathrm{BID}, i}\right)
\end{aligned}
$$

The spread $S_{t}(N)$ is constant if $N \leq N^{-}$and is an increasing function if $N>N^{-}$. If $N>N^{+}$, the relationship is linear.

From a theoretical point of view, the relationship between the spread and the notional is more complicated. It is generally admitted that the spread remains flat up to $N^{-}$and the marginal impact increases linearly up to $N^{\star}$ (Acerbi and Szekeres, 2013). $N^{\star}$ measures the market depth of the ETF, which is the maximum order size that may be instantly executable. By construction, we have $N^{\star}>N^{+}$. If $N>N^{\star}$, it is unrealistic to execute the order size, meaning that the spread is infinite (see the second panel in Figure 6). We adopt here a pragmatic approach by considering the empirical relationship if $N \leq N^{+}$and the linear assumption if $N>N^{+}$. However, we consider that the slope of the linear relationship 
is equal to $S\left(N^{+}\right) / N^{+}$, which is generally higher than the average empirical slope ${ }^{13}$. The idea is to obtain a conservative measure of liquidity by penalising ETFs with low order book volumes.

Daily analysis In Figure 7, we report the evolution for the liquidity spread of three ETF providers, when the benchmark is the EURO STOXX 50 index and for different values of the notional $N$ (expressed in $\mathrm{M} €$ ). We notice that this spread changes with respect to market liquidity. The second provider has two ETF funds in Europe, which explains the distinction we make between \#2 (a) and \#2 (b). Curiously, we note that the difference between the spreads of these two ETFs is very large. We have shown the boxplot for each tracker and each notional value in Figure 8. We observe that the spread of ETF \#3 is less sensitive to the notional than those of ETFs \#1 and \#2. Finally, we compute the median liquidity spread for 2012 in Table 1. We verify that the differences increase when the notional increases. This means that the choice of an ETF becomes essential when the investor wants to trade a significant notional.

Figure 7: Evolution of the liquidity spread (EURO STOXX 50)

$\# 1$
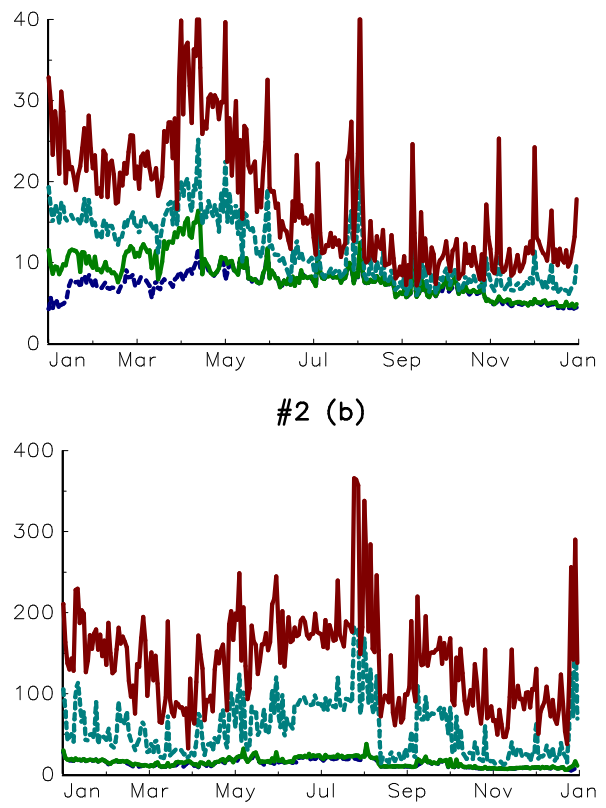

\#2 (a)
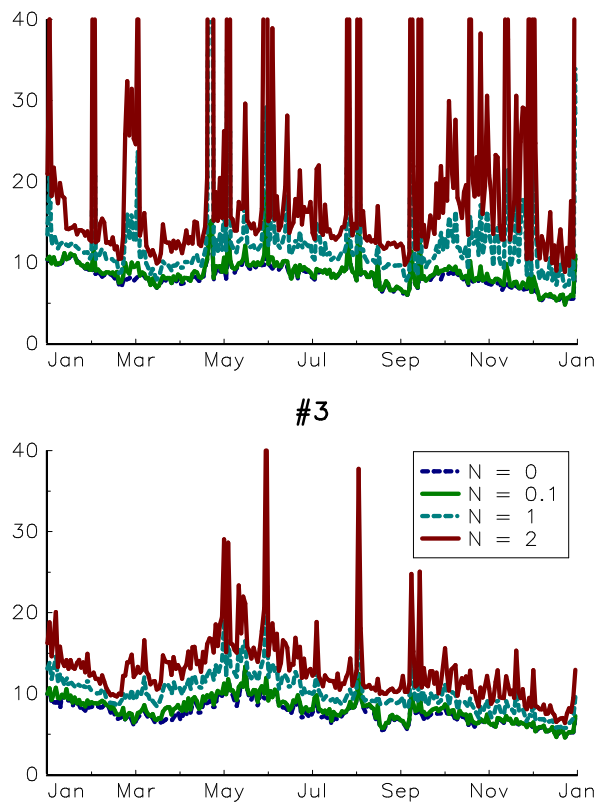

In Table 1, we indicate the median spread for different ETF providers and several values of the notional $N$. The ETF providers \#1, \#2, \#3 and \#4 offer similar liquidity conditions, which is not the case for the ETF provider \#8. We also notice that when a provider manages two similar ETFs ${ }^{14}$, we generally observe significant differences. This is the case for ETF provider \#2. If we consider the S\&P 500 indexation, we can split the ETF providers into four categories (Table 2). ETF manager \#3 provides a spread lower than 10 bps even when the notional is $2 \mathrm{M} €$. The spread of ETF managers \#2, \#4 and \#8 increases with the

\footnotetext{
${ }^{13}$ It corresponds to the estimated slope for $N^{-}<N<N^{+}$.

${ }^{14}$ They differ because of their domicile (Luxembourg, Ireland, Germany, France, UK, etc.).
} 
Figure 8: Boxplot of the liquidity spread (EURO STOXX 50)
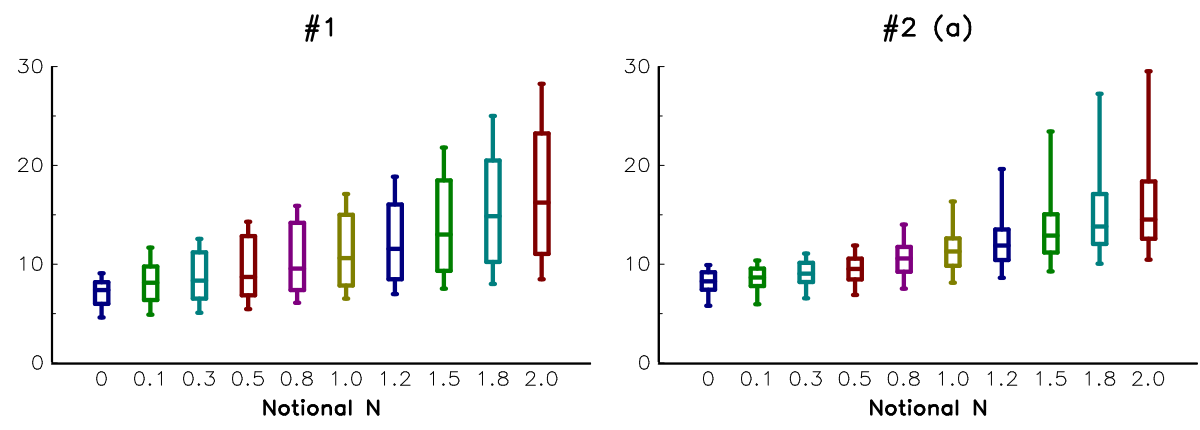

\#2 (b)

\#3
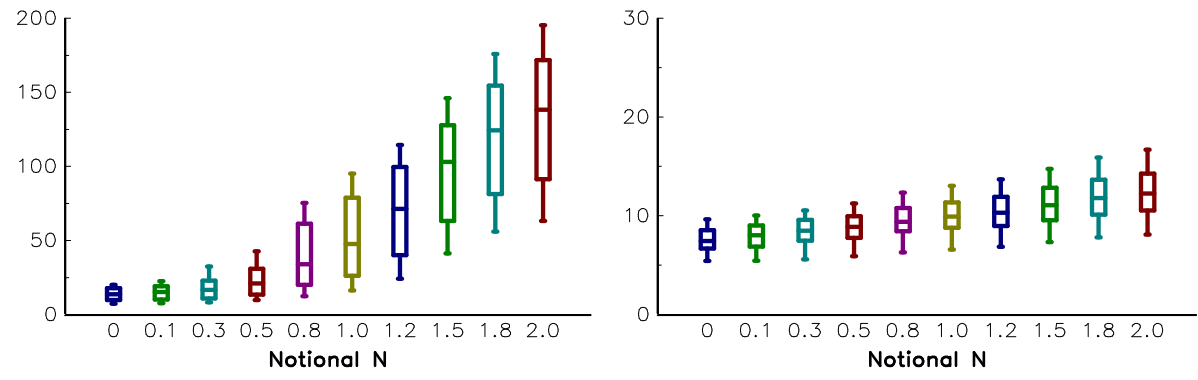

Table 1: Median liquidity spread (EURO STOXX 50)

\begin{tabular}{|c|c|c|c|c|c|c|c|}
\hline$N($ in $\mathrm{M} €)$ & $\# 1$ & (a) & 2 & (a) & $\begin{array}{l}3 \\
\text { (b) }\end{array}$ & & \# \\
\hline 0.0 & 7 & 8 & 14 & 7 & 7 & 7 & \\
\hline 0.1 & 8 & 9 & 15 & 8 & 8 & 7 & \\
\hline 0.3 & 8 & 9 & 17 & 8 & 8 & 8 & \\
\hline 0.5 & 9 & 10 & 21 & 8 & 9 & 9 & \\
\hline 0.8 & 10 & 11 & 34 & 8 & 9 & 10 & \\
\hline 1.0 & 11 & 11 & 48 & 9 & 10 & 10 & I \\
\hline 1.3 & 12 & 12 & 71 & 9 & 10 & 11 & $\begin{array}{l}1 \\
1\end{array}$ \\
\hline 1.5 & 13 & 13 & 103 & 10 & 11 & 11 & \\
\hline 1.8 & 15 & 14 & 126 & 10 & 12 & 12 & 12 \\
\hline 2.0 & 16 & 15 & 140 & 10 & 12 & 13 & 11 \\
\hline
\end{tabular}


Table 2: Median liquidity spread (S\&P 500)

\begin{tabular}{|c|rrrrrrr|}
\hline$N($ in M $€$ ) & $\# 1$ & $\# 2$ & $\# 3$ & $\# 4$ & $\# 7$ & $\# 8$ & $\# 9$ \\
\hline 0.0 & 10 & 9 & 4 & 9 & 10 & 14 & 29 \\
0.1 & 13 & 11 & 5 & 9 & 11 & 14 & 29 \\
0.3 & 15 & 12 & 5 & 9 & 12 & 14 & 32 \\
0.5 & 18 & 14 & 6 & 10 & 14 & 16 & 57 \\
0.8 & 22 & 15 & 6 & 13 & 18 & 17 & 103 \\
1.0 & 25 & 17 & 6 & 16 & 23 & 18 & 137 \\
1.3 & 30 & 19 & 7 & 17 & 30 & 18 & 158 \\
1.5 & 37 & 22 & 7 & 19 & 43 & 19 & 197 \\
1.8 & 45 & 24 & 7 & 22 & 54 & 19 & 236 \\
2.0 & 50 & 26 & 7 & 23 & 61 & 20 & 262 \\
\hline
\end{tabular}

Table 3: Median liquidity spread (MSCI WORLD)

\begin{tabular}{|c|rrrrrrrrr|}
\hline$N$ (in M€) & $\# 1$ & $\# 2$ & $\# 3$ & $\# 4$ & $\# 5$ & $\# 6$ & $\# 7$ & $\# 8$ & $\# 9$ \\
\hline 0.0 & 12 & 9 & 12 & 9 & 13 & 40 & 32 & 22 & 35 \\
0.1 & 13 & 11 & 13 & 9 & 17 & 41 & 33 & 23 & 35 \\
0.3 & 14 & 11 & 13 & 10 & 19 & 45 & 36 & 23 & 62 \\
0.5 & 16 & 12 & 14 & 10 & 22 & 48 & 42 & 24 & 91 \\
0.8 & 23 & 13 & 15 & 13 & 25 & 51 & 51 & 25 & 145 \\
1.0 & 26 & 14 & 15 & 14 & 27 & 69 & 61 & 26 & 181 \\
1.3 & 30 & 15 & 16 & 15 & 30 & 98 & 74 & 27 & 218 \\
1.5 & 36 & 16 & 17 & 16 & 73 & 136 & 92 & 29 & 272 \\
1.8 & 43 & 17 & 17 & 19 & 111 & 172 & 110 & 32 & 326 \\
2.0 & 48 & 18 & 18 & 20 & 130 & 194 & 123 & 33 & 363 \\
\hline
\end{tabular}

Table 4: Median liquidity spread (IBOXX EUR LIQUID)

\begin{tabular}{|c|cccc|}
\hline$N($ in $\mathrm{€} €)$ & $\# 1$ & $\# 3$ & $\# 4$ & $\# 9$ \\
\hline 0.0 & 24 & 10 & 13 & 20 \\
0.1 & 26 & 12 & 19 & 20 \\
0.3 & 27 & 14 & 22 & 20 \\
0.5 & 28 & 15 & 24 & 20 \\
0.8 & 28 & 17 & 25 & 20 \\
1.0 & 28 & 19 & 27 & 23 \\
1.3 & 28 & 23 & 27 & 28 \\
1.5 & 29 & 30 & 28 & 34 \\
1.8 & 29 & 38 & 29 & 41 \\
2.0 & 29 & 43 & 29 & 46 \\
\hline
\end{tabular}


notional, but remains at an acceptable level (lower than $25 \mathrm{bps}$ ). For ETF managers \#1 and \#7 the spread is very sensitive to the notional $N$. Finally, the spread of ETF manager \#9 is off the scale because of the lack of limit orders. We obtain similar patterns if we consider the MSCI World indexation (Table 3).

Let us now consider fixed income instruments. In Table 4, we report the results obtained for the Markit iBoxx EUR Liquid Corporates index. It follows that the best spreads are larger than those observed on the equity side. This is the case for the best limit, but also when the notional increases. For instance, the spread of ETF providers \#1 and \#4 is 29 bps when the notional is $2 \mathrm{M} €$.

Intraday analysis In the previous paragraph, we compute the daily spread according to Equation (3). This formula is also valid for a given period $\left[t_{\text {begin }}, t_{\text {end }}\right]$ :

$$
S(N)=\frac{\sum_{t_{j}=t_{\text {begin }}}^{t_{\text {end }}} S_{t_{j}}(N)\left(t_{j+1}-t_{j}\right)}{\sum_{t_{j}=t_{\text {begin }}}^{t_{\text {end }}}\left(t_{j+1}-t_{j}\right)}
$$

In what follows, we compute the intraday liquidity spread for a period of 30 minutes and a notional of $1 \mathrm{M} €$. In Figure 25 in Appendix $\mathrm{C}$ on page 33, we report the boxplot of intraday spreads from 9:00 to 17:30 in the case of the EURO STOXX 50 index. We notice that the distribution of the spread is not homogeneous during the day. Indeed, the spread is more volatile during three periods:

- At the opening of the European market between 9:00 and 9:30.

- Before the opening of the US market between 14:00 and 15:00.

- After the opening of the US market between 15:30 and 16:30.

We observe this phenomenon for all equity ETFs. For instance, we report the boxplot of the intraday spread for the MSCI World index in Figure 26 in Appendix C on page 33. These volatility peaks are explained by the news announcement. But curiously, they are specific to the equity market and we do not observe the phenomenon for fixed income ETFs ${ }^{15}$.

\subsubsection{Computing the liquidity notional}

Definition Following Degryse et al. (2011), we define the liquidity notional as follows:

$$
N_{t}(S)=\frac{N_{t}^{\mathrm{BID}}\left(\frac{S}{2}\right)+N_{t}^{\mathrm{ASK}}\left(\frac{S}{2}\right)}{2}
$$

with:

$$
\begin{aligned}
N_{t}^{\mathrm{BID}}(S) & =\sum_{i=1}^{5} P_{t}^{\mathrm{BID}, i} Q_{t}^{\mathrm{BID}, i} \mathbb{1}\left\{P_{t}^{\mathrm{BID}, i} \geq(1-S) \cdot P_{t}^{\mathrm{MID}}\right\} \\
N_{t}^{\mathrm{ASK}}(S) & =\sum_{i=1}^{5} P_{t}^{\mathrm{ASK}, i} Q_{t}^{\mathrm{ASK}, i} \mathbb{1}\left\{P_{t}^{\mathrm{ASK}, i} \leq(1+S) \cdot P_{t}^{\mathrm{MID}}\right\}
\end{aligned}
$$

$N_{t}(S)$ represents the notional that can be traded immediately without causing an updated spread larger than $S$. Therefore, combining the spread and volume of limit order books is an alternative method to the liquidity spread.

\footnotetext{
${ }^{15}$ This is confirmed by the boxplot of Markit iBoxx EUR Liquid Corporates ETFs in Figure 27 in Appendix $\mathrm{C}$ on page 34 .
} 
If we consider the previous example, we obtain the function $N_{t}(S)$ given in Figure 9. For instance, if we accept a spread equal to $10 \mathrm{bps}$, we obtain a notional of $4.4 \mathrm{M} €$. Even if the liquidity measure $N_{t}(S)$ is not the inverse function of the liquidity measure $S_{t}(N)$, the two measures are highly correlated.

Figure 9: Evolution of the liquidity notional with respect to the spread

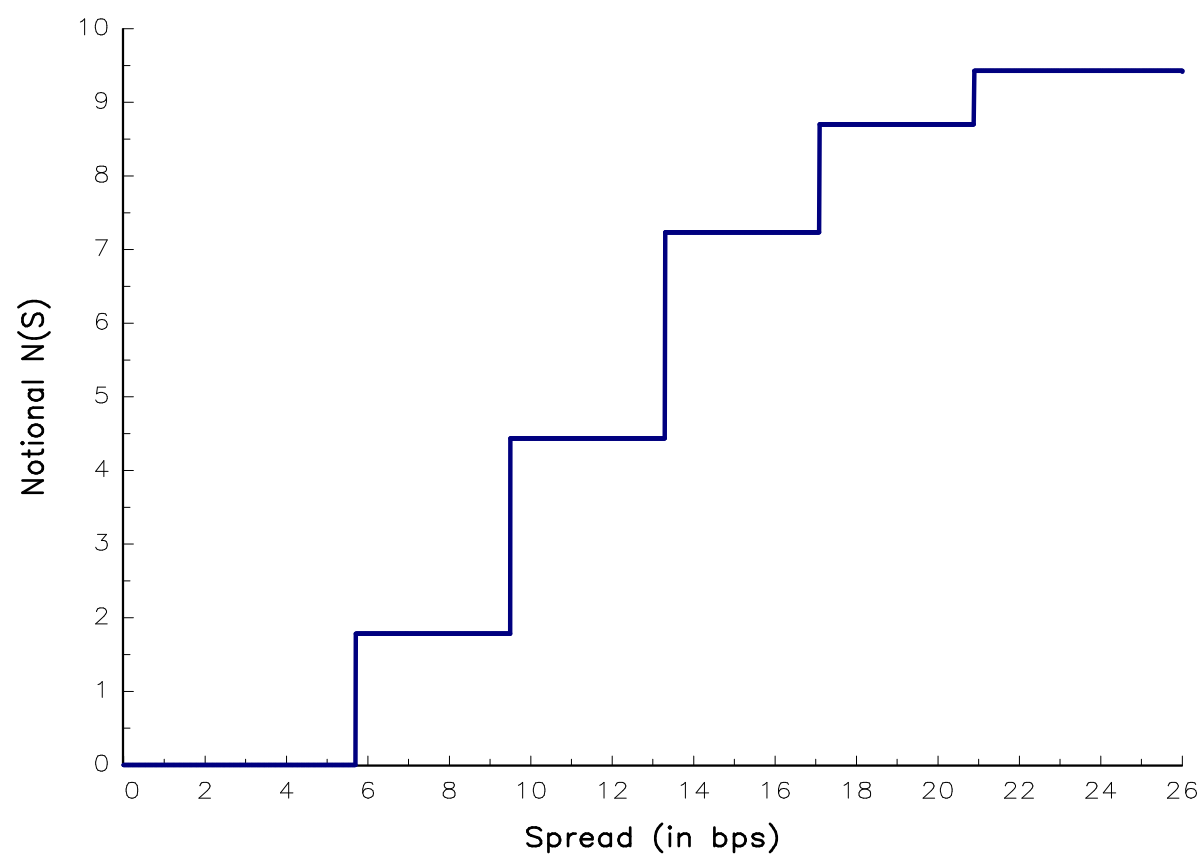

Daily analysis In a similar way to Equation (3), the daily liquidity notional $N(S)$ corresponds to the intraday liquidity notional weighted by the duration between two ticks:

$$
N(S)=\frac{\sum_{t_{j}=\text { open }}^{\text {close }} N_{t_{j}}(S)\left(t_{j+1}-t_{j}\right)}{\sum_{t_{j}=\text { open }}^{\text {close }}\left(t_{j+1}-t_{j}\right)}
$$

In Figure 10, we report the evolution of the liquidity notional (expressed in $\mathrm{M} €$ ) of four EURO STOXX 50 ETFs for different values of the spread. The small differences of 30 bps and 50 bps are due to the limited available order sizes. The liquidity notional is more sensitive to a lower spread (less than $20 \mathrm{bps}$ ). Besides, we notice that the liquidity notional increases overall during 2012 but undergoes two sharp falls in August and November. We also observe different behaviours at the end of 2012. For instance, the liquidity notional increases for ETF \#4, whereas it decreases for ETFs \#1, \#2 and \#3. The corresponding boxplots are reported in Figure 11. These results are consistent with those obtained in Figure 8.

Intraday analysis The calculation of the intraday liquidity notional is similar to that of the intraday liquidity spread. We present the boxplot of these statistics in Figure 28 on page 34. We notice that the distribution of the liquidity notional is more homogeneous during the day than the distribution of the liquidity spread. This implies that the liquidity notional is 
Figure 10: Evolution of the liquidity notional (EURO STOXX 50)
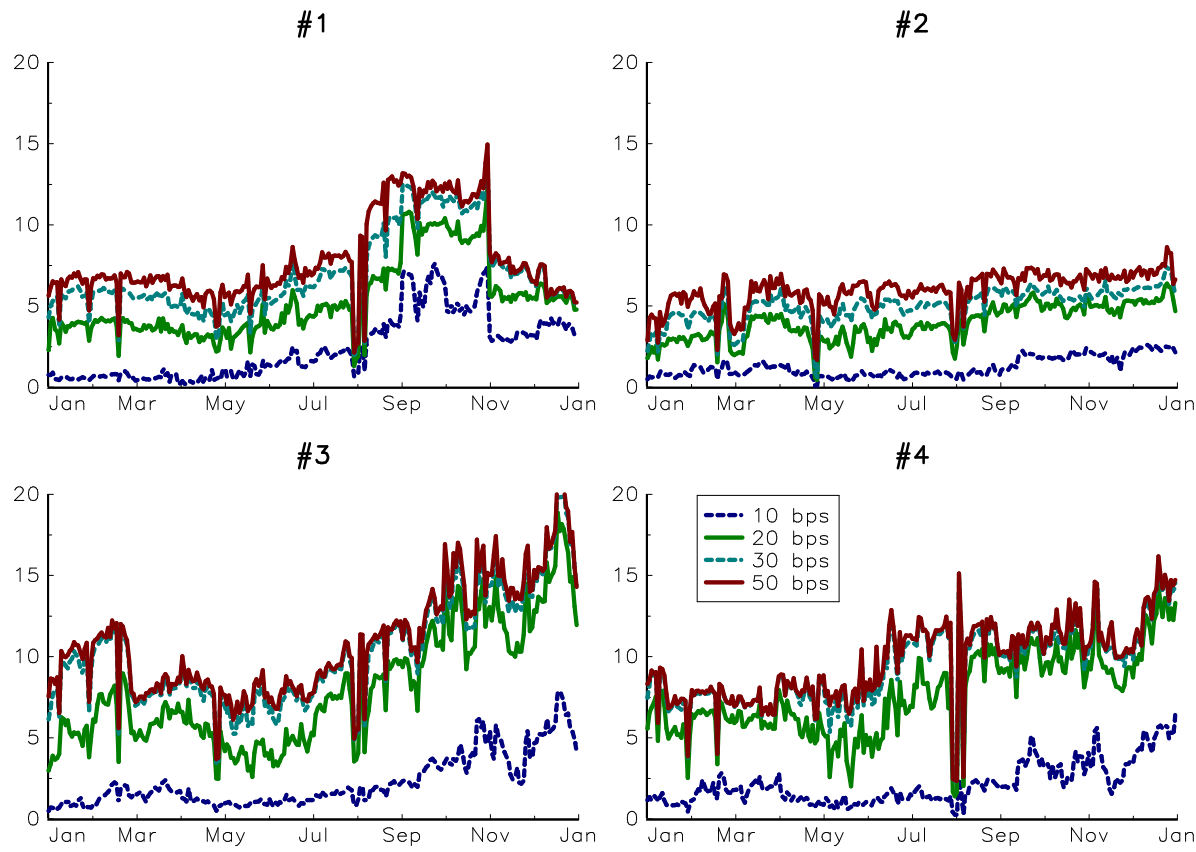

Figure 11: Boxplot of the liquidity notional (EURO STOXX 50)

$\# 1$
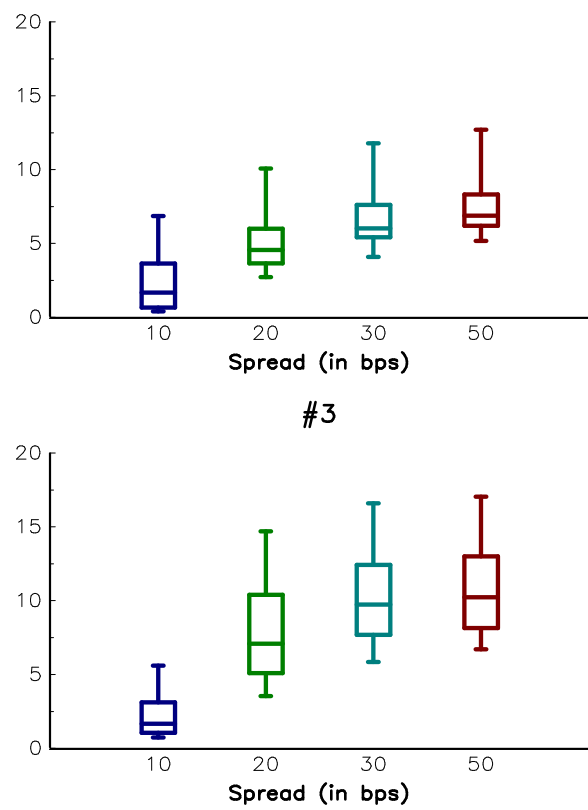

\#2
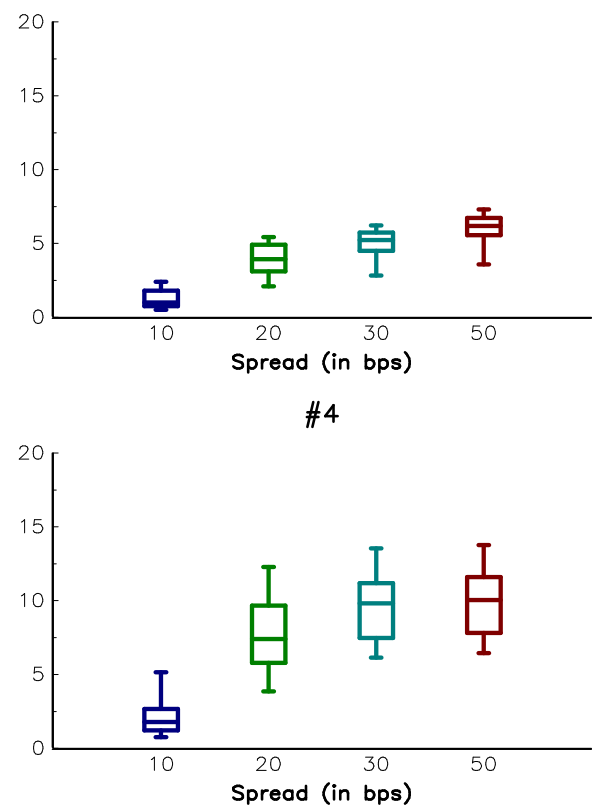
Measuring the Liquidity of ETFs: An Application to the European Market

a measure that is less sensitive and less reactive to the updates of the order book than the liquidity spread.

\subsection{On the interconnection between spread, volume and liquidity}

In Tables 5 and 6 , we present the cross-correlations expressed in $\%$ between the different liquidity measures: the trading volume $Q$, the best limit volume $Q^{\mathrm{MEAN}, 1}$, the liquidity spread $S(N)$ (for $N=1 \mathrm{M} €$ ) and the liquidity notional $N(S)$ (for $S=30 \mathrm{bps}$ ). We consider the cross-correlations between the level of these measures (Table 5) and between their variations (Table 6). We observe that the liquidity spread is negatively correlated with the liquidity notional and the best limit volume. Curiously, the correlation between the liquidity spread and trading volume is not significant. We also notice that these correlations are not very stable and depend on the study period. These results suggest that the relationships between the different liquidity measures, if they exist, are not linear.

Table 5: Correlation between liquidity measures (EURO STOXX 50)

\begin{tabular}{|c|c|c|c|c|c|c|c|}
\hline \multirow{2}{*}{ Frequency } & \multirow{2}{*}{ ETF } & \multicolumn{3}{|c|}{$O$} & \multicolumn{2}{|c|}{$Q^{\mathrm{MEAN}, 1}$} & \multirow{2}{*}{$\begin{array}{l}S(N) \\
N(S)\end{array}$} \\
\hline & & $Q^{\mathrm{MEAN}, 1}$ & $S(N)$ & $N(S)$ & $S(N)$ & $N(S)$ & \\
\hline \multirow{3}{*}{ Daily } & $\# 1$ & 18 & -16 & 11 & -58 & 92 & -64 \\
\hline & \#2 & -2 & -5 & -10 & -28 & 51 & -44 \\
\hline & $\# 3$ & 14 & -9 & 14 & -18 & 92 & -19 \\
\hline \multirow{5}{*}{ Intraday } & $\# 4$ & 9 & 2 & 12 & -56 & 95 & -59 \\
\hline & $\# 1$ & 10 & 9 & 5 & $-\overline{-3} 7^{-}$ & $\overline{9} 0$ & $-\overline{4} 6$ \\
\hline & \#2 & 0 & 0 & -6 & -23 & 49 & -28 \\
\hline & \#3 & 5 & 0 & 5 & -4 & 89 & -4 \\
\hline & \#4 & 1 & 8 & 1 & -17 & 99 & -21 \\
\hline
\end{tabular}

Table 6: Correlation between variations of liquidity measures (EURO STOXX 50)

\begin{tabular}{|c|c|c|c|c|c|c|c|}
\hline \multirow{2}{*}{ Frequency } & \multirow{2}{*}{ ETF } & \multicolumn{3}{|c|}{$Q$} & \multicolumn{2}{|c|}{$Q^{\mathrm{MEAN}, 1}$} & \multirow{2}{*}{$\begin{array}{l}S(N) \\
N(S)\end{array}$} \\
\hline & & $Q^{\mathrm{MEAN}, 1}$ & $S(N)$ & $N(S)$ & $S(N)$ & $N(S)$ & \\
\hline \multirow{4}{*}{ Daily } & $\# 1$ & 21 & 6 & 15 & -23 & 87 & -24 \\
\hline & $\# 2$ & 17 & -6 & 17 & -10 & 58 & -46 \\
\hline & \#3 & 11 & -7 & 15 & -41 & 71 & -44 \\
\hline & \#4 & 19 & -2 & 22 & -48 & 98 & -51 \\
\hline \multirow{4}{*}{ Intraday } & $\# 1$ & -4 & $1 \overline{8}$ & -6 & -26 & 50 & $-\overline{4} 2$ \\
\hline & $\# 2$ & 3 & 4 & -1 & -17 & 38 & -38 \\
\hline & \#3 & -5 & 1 & -5 & -1 & 46 & 0 \\
\hline & $\# 4$ & 0 & 6 & 0 & 0 & 97 & -1 \\
\hline
\end{tabular}

In Figure 12, we report the scatterplot of the intraday spread versus the trading volume for the EURO STOXX 50 ETF \#1. We note that most of the points are concentrated in a region bounded by an hyperbolic curve. This property is not specific to this particular ETF: it is shared by all the ETFs we have studied.

Let $S_{j}$ and $Q_{j}$ be the spread and trading volume for the $j^{\text {th }}$ period of 30 minutes. We consider $n$ observations. We search the upper bound curve satisfying the following 
Figure 12: Scatterplot of intraday spread versus trading volume (EURO STOXX 50 ETF \#1)

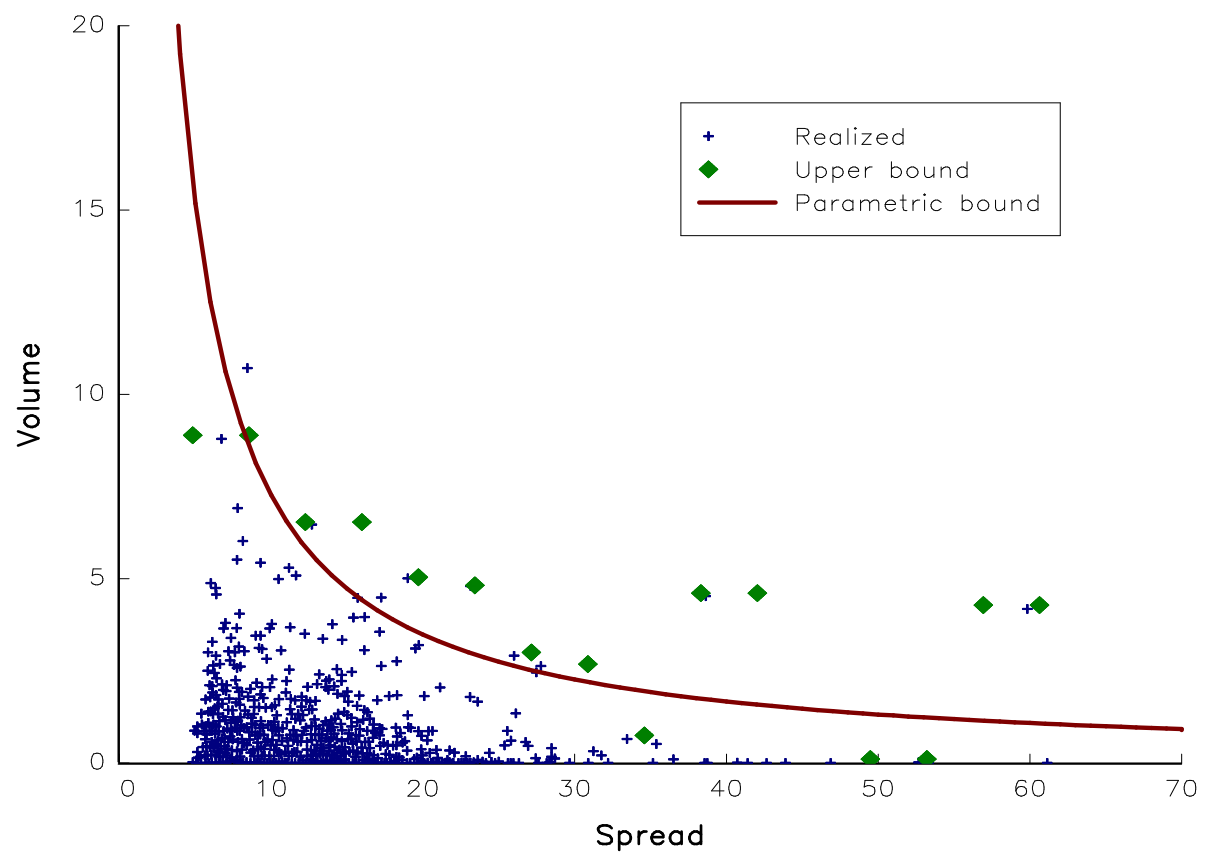

optimization problem:

$$
\hat{f}=\arg \min \int_{0}^{\infty} f(s) \mathrm{d} s-\lambda \frac{\operatorname{card}\left\{V_{j}: V_{j} \leq f\left(S_{j}\right)\right\}}{n}
$$

where $\operatorname{card}\left\{V_{j}: V_{j} \leq f\left(S_{j}\right)\right\}$ denotes the number of points below the curve $f$ and $\lambda$ is the coefficient which controls the number of points above the curve. The above optimisation is not easy to solve if we consider $\mathcal{C}^{1}$ functions. However, it is less difficult when we look for a piecewise constant function. For instance, the calibrated piecewise constant function $\hat{f}$ with $\lambda=5$ is indicated by green diamonds in Figure 12. We then postulate that the theoretical curve is defined by $f(s)=\alpha s^{\beta}$ where $\alpha$ and $\beta$ are two parameters to estimate. The parameters can be estimated using the method of least squares ${ }^{16}$. The previous analysis allow us to define a theoretical liquidity measure:

$$
\mathcal{L}^{\star}=\int_{0}^{\infty} f(s) g(s) \mathrm{d} s
$$

and an empirical liquidity measure:

$$
\hat{\mathcal{L}}=\int_{0}^{\infty} \hat{f}(s) \hat{g}(s) \mathrm{d} s
$$

where $g(s)$ is the theoretical distribution of the spread and $\hat{g}(s)$ is the empirical distribution of the spread. In Figure 13, we illustrate the computation of $\hat{\mathcal{L}}$. It combines two informations:

1. the empirical relationship between the spread and the trading volume;

2. the empirical distribution of the spread. 
Figure 13: Illustration of the liquidity measure computation

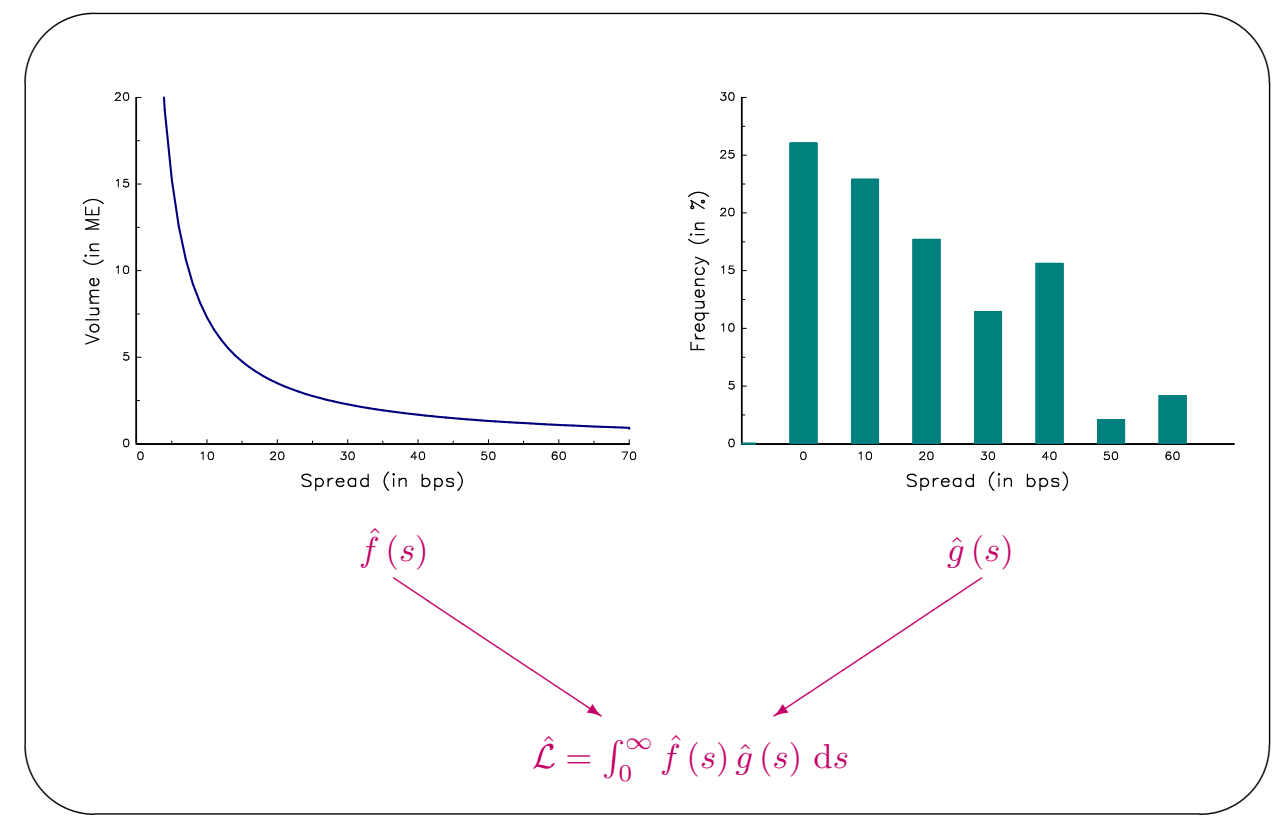

Therefore, we can define the liquidity preference ordering $\succ$ as follows:

Proposition 2 ETF \#2 is preferable to ETF \#1 if and only if the liquidity measure of ETF \#2 is greater than the liquidity measure of ETF \#1:

$$
\# 2 \succ \# 1 \Leftrightarrow \hat{\mathcal{L}}(\# 2)>\hat{\mathcal{L}}(\# 1)
$$

In Figure 14, the trading volume of ETF \#2 is larger than the trading volume of ETF \#1 for a given spread. Moreover, ETF \#2 presents lower spreads than ETF \#1. This is why we prefer ETF \#2, which has a higher liquidity measure.

The empirical liquidity measure for the ETFs on EURO STOXX 50, S\&P 500, MSCI WORLD and Markit iBoxx EUR Liquid Corporates are reported respectively in Tables 7, 8,9 and 10. The median spread ${ }^{17} S(N)$ (expressed in bps) corresponds to a notional of 1 $\mathrm{M} €$. The volume $Q$ (expressed in $\mathrm{M} €$ ) corresponds to the average trading volume over 30 minutes. By comparing the empirical liquidity measure $\hat{\mathcal{L}}$ with classical measures such as $S(N)$ and $Q$, we find that the liquidity measure $\hat{\mathcal{L}}$ makes sense since it takes into account the liquidity spread as well as the trading volume. We therefore obtain the following:

1. The liquidity measure $\hat{\mathcal{L}}$ determines the market capacity, whereas the trading volumes are statistical realisations under the capacity limit of the market. In other words, the liquidity spread is an endogenous factor, but the trading volume is an exogenous factor used to determine market liquidity.

2. In the case where there are few transactions, it will be especially difficult to determine the empirical liquidity measure $\hat{\mathcal{L}}$. It will therefore be more practical to evaluate the liquidity using the liquidity spread $S(N)$.

\footnotetext{
${ }^{16}$ For example, we obtain $\hat{\alpha}=83.8$ and $\hat{\beta}=-1.06$ for the EURO STOXX 50 ETF \#1.

${ }^{17}$ We observe some small differences compared with the median spread computed in Tables $1-4$, because the sampling period is not the same (daily versus 30 minutes).
} 
Figure 14: The preference ordering: $\# 2 \succ \# 1 \Leftrightarrow \hat{\mathcal{L}}(\# 2)>\hat{\mathcal{L}}(\# 1)$

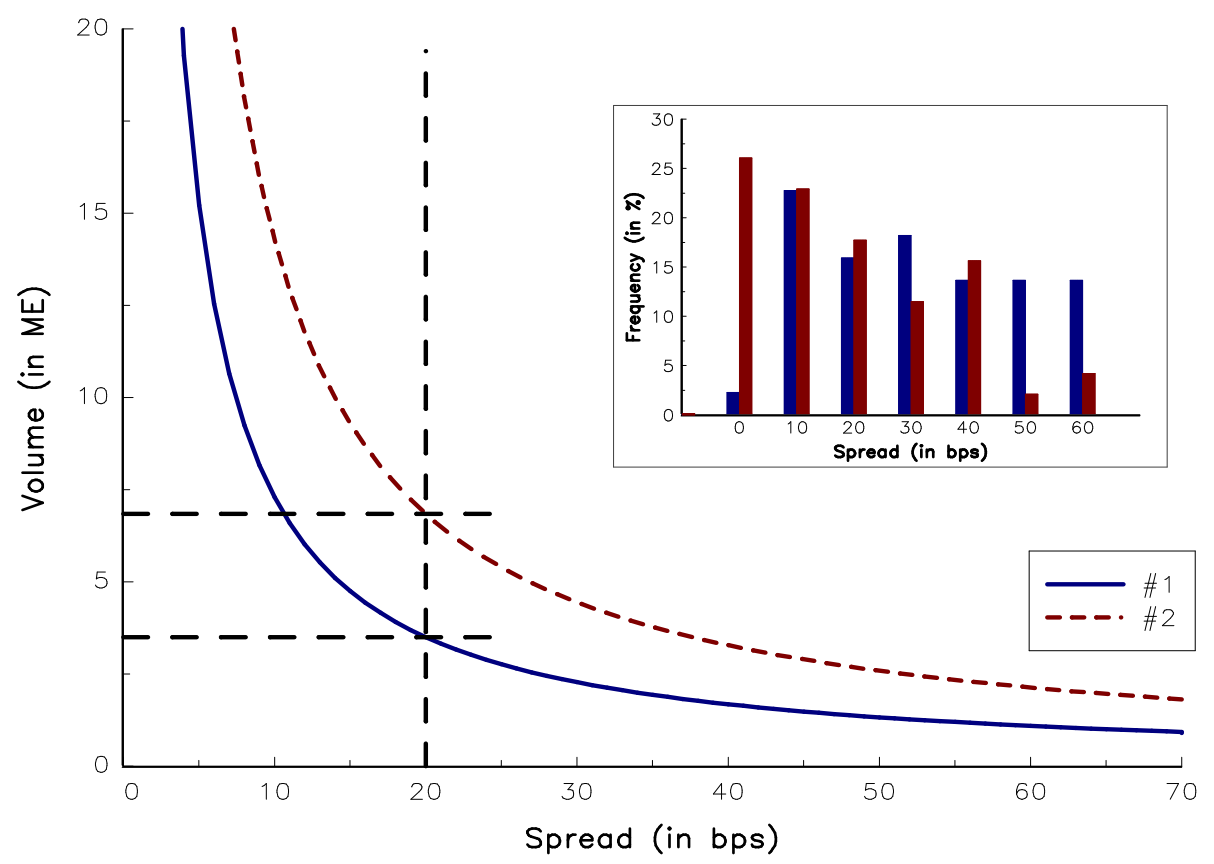

Table 7: Liquidity measures (EURO STOXX 50)

\begin{tabular}{|l|rrr|}
\hline ETF & \multicolumn{1}{|c}{$S(N)$} & \multicolumn{1}{c|}{$Q$} & \multicolumn{1}{c|}{$\hat{\mathcal{L}}$} \\
\hline$\# 1$ & 10.1 & 163 & 16.5 \\
$\# 2(\mathrm{a})$ & 10.4 & 235 & 8.8 \\
$\# 2$ (b) & 36.6 & 144 & 8.0 \\
$\# 3(\mathrm{a})$ & 8.6 & 864 & 35.3 \\
$\# 3$ (b) & 9.5 & 969 & 48.8 \\
$\# 4$ & 9.4 & 825 & 29.9 \\
$\# 8$ & 17.3 & 27 & 4.5 \\
\hline
\end{tabular}

Table 8: Liquidity measures (S\&P 500)

\begin{tabular}{|l|rrr|}
\hline ETF & $S(N)$ & \multicolumn{1}{|c|}{$Q$} & \multicolumn{1}{c}{$\hat{\mathcal{L}}$} \\
\hline$\# 1(\mathrm{a})$ & 23.6 & 21 & 2.9 \\
$\# 1$ (b) & 57.8 & 5 & 0.1 \\
$\# 2$ & 14.8 & 84 & 13.7 \\
$\# 3$ (a) & 5.6 & 1194 & 68.0 \\
$\# 3$ (b) & 6.8 & 320 & 48.7 \\
$\# 4(\mathrm{a})$ & 14.1 & 34 & 5.4 \\
$\# 4$ (b) & 131.3 & 11 & 0.0 \\
$\# 6$ & 19.0 & 62 & 6.4 \\
$\# 7(\mathrm{a})$ & 18.9 & 54 & 3.4 \\
$\# 7$ (b) & 189.0 & 3 & 0.0 \\
$\# 8(\mathrm{a})$ & 14.7 & 22 & 2.4 \\
$\# 8$ (b) & 26.6 & 3 & 1.5 \\
$\# 9$ & 122.6 & 3 & 0.0 \\
\hline
\end{tabular}


Table 9: Liquidity measures (MSCI WORLD)

\begin{tabular}{|l|rrr|}
\hline ETF & $S(N)$ & \multicolumn{1}{c|}{$Q$} & \multicolumn{1}{c|}{$\hat{\mathcal{L}}$} \\
\hline$\# 1(\mathrm{a})$ & 24.1 & 20 & 4.7 \\
$\# 1$ (b) & 34.2 & 10 & 0.3 \\
$\# 2$ & 12.7 & 427 & 24.5 \\
$\# 3(\mathrm{a})$ & 13.0 & 571 & 10.9 \\
$\# 3(\mathrm{~b})$ & 14.7 & 157 & 9.5 \\
$\# 4(\mathrm{a})$ & 12.0 & 151 & 10.5 \\
$\# 4$ (b) & 21.7 & 13 & 2.8 \\
$\# 5$ & 11.1 & 16 & 2.9 \\
$\# 6$ & 61.0 & 6 & 0.0 \\
$\# 7$ & 59.4 & 13 & 0.0 \\
$\# 8$ & 23.7 & 30 & 8.9 \\
$\# 9$ & 163.0 & 0 & 0.0 \\
\hline
\end{tabular}

Table 10: Liquidity measures (IBOXX EUR LIQUID)

\begin{tabular}{|l|crr|}
\hline ETF & $S(N)$ & \multicolumn{1}{c|}{$Q$} & \multicolumn{1}{c|}{$\hat{\mathcal{L}}$} \\
\hline$\# 1$ & 28.1 & 15 & 1.1 \\
$\# 3(\mathrm{a})$ & 18.6 & 108 & 8.7 \\
$\# 3(\mathrm{~b})$ & 23.8 & 215 & 13.8 \\
$\# 4$ & 25.7 & 53 & 3.6 \\
$\# 9$ & 22.7 & 2 & 0.3 \\
\hline
\end{tabular}

\section{Measuring the relative liquidity of ETFs}

In the previous section, we proposed different absolute measures to assess the liquidity of an ETF. We call them absolute because we do not refer explicitly to the liquidity of the underlying index. In this section, we investigate the relative liquidity, by combining both the liquidity of the ETF and the liquidity of the underlying index.

\subsection{Trading volume}

The trading volume of a security for a given period is defined as the sum of the traded volumes multiplied by the corresponding market prices. Similarly, if we want to compute the trading volume of an index $T_{t}^{\text {Index }}$, we add the trading volumes of the different assets that compose the index. Moreover, we can add the trading volumes of the different ETFs that track the index in order to obtain an aggregated trading volume $V_{t}^{\mathrm{ETF}}$.

In Figure 15, we report the scatterplot of the index trading volume (expressed in $\mathrm{B} €$ ) versus the ETF trading volume (expressed in $\mathrm{M} €$ ). We notice that the magnitude is not the same. However, we observe that there is a significant relationship between the two trading volumes. If we consider the futures market instead of the cash market, we obtain similar results (see Figure 16). However, we notice the following differences:

- the ETF trading volume is more closely correlated to the index trading volume than to the futures trading volume in the case of the CAC 40 and DAX indices;

- the ETF trading volume is more closely correlated to the futures trading volumes than to the index trading volumes in the case of the S\&P 500 index; 
Figure 15: Scatterplot of the index trading volume versus the ETF trading volume
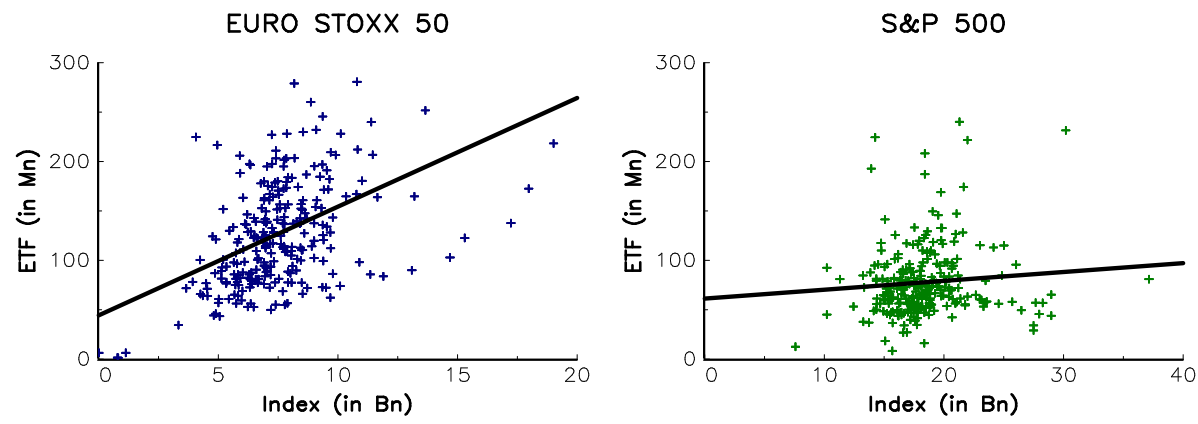

CAC 40
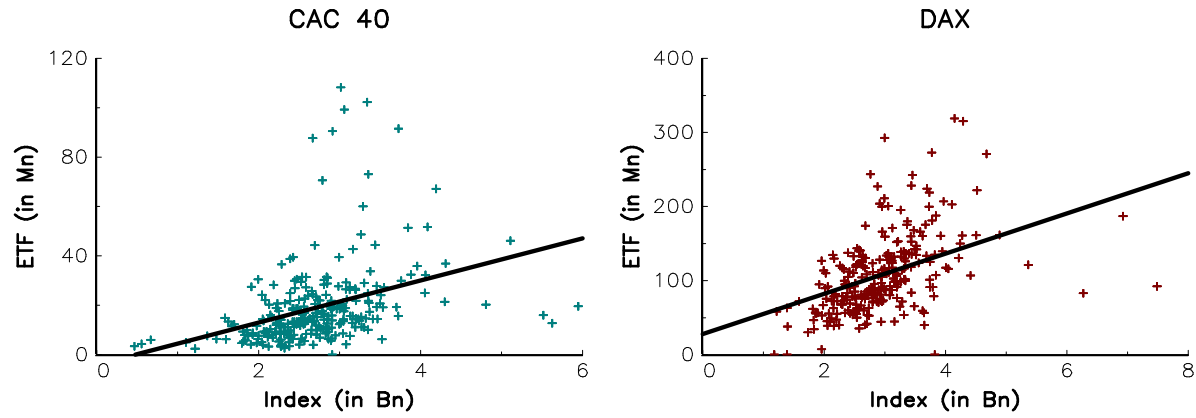

Figure 16: Scatterplot of the futures trading volume versus the ETF trading volume
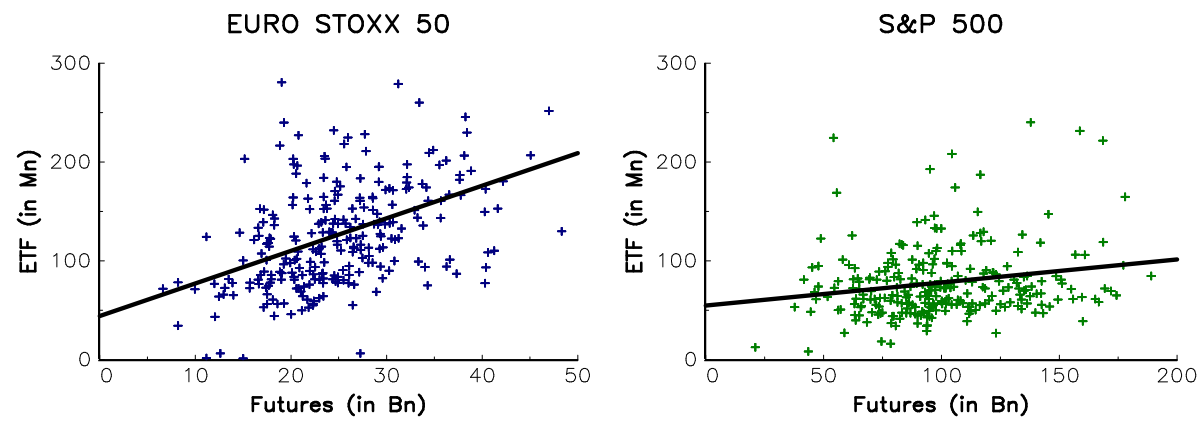

CAC 40
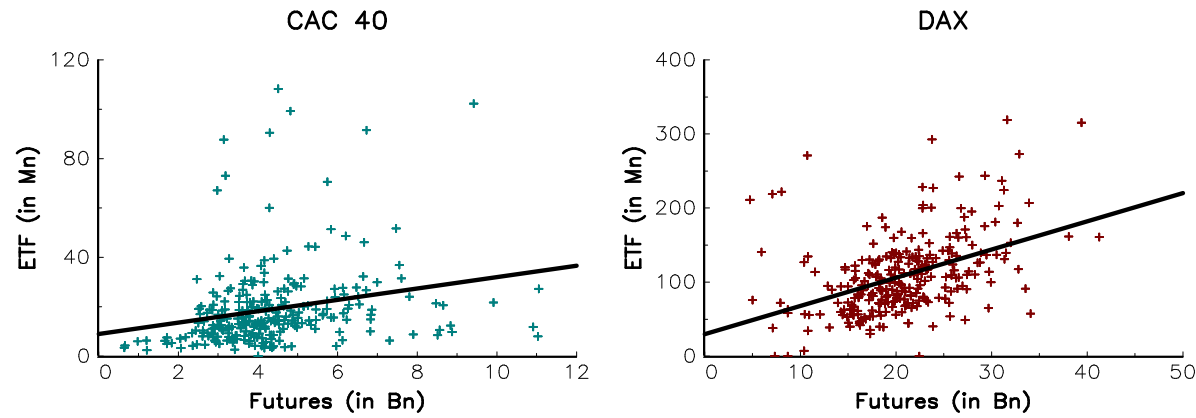
- in the case of the EURO STOXX 50 index, there is no significant difference between the correlation with the index trading volume and the correlation with the futures trading volume.

In Table 11, we report statistics for the ratio between the ETF trading volume and the index (or futures) trading volume:

$$
\mathcal{V} \mathcal{R}_{t}=\frac{V_{t}^{\mathrm{ETF}}}{V_{t}^{\text {Index }}}
$$

The figures are very low. For instance, the ETF trading volume represents less than $4 \%$ of the index or futures trading volume if we consider the median.

Table 11: Statistics of the trading volume ratio $\mathcal{V} \mathcal{R}_{t}$

\begin{tabular}{|c|l|cccc|}
\hline Quantile & & EURO STOXX 50 & CAC 40 & DAX & S\&P 500 \\
\hline \multirow{2}{*}{$50 \%$} & Index & $1.64 \%$ & $0.56 \%$ & $3.45 \%$ & $0.39 \%$ \\
& Futures & $0.48 \%$ & $0.36 \%$ & $0.49 \%$ & $0.07 \%$ \\
\hline \multirow{2}{*}{$95 \%$} & Index & $2.89 \%$ & $1.59 \%$ & $6.44 \%$ & $0.78 \%$ \\
& Futures & $0.92 \%$ & $0.98 \%$ & $0.98 \%$ & $0.16 \%$ \\
\hline
\end{tabular}

\subsection{Intraday spread}

Although some studies have looked at the impact of ETFs on the liquidity of their underlyings (Deville et al., 2011; De Winne et al., 2013), little analysis has been done on the liquidity relationship between ETFs and their underlyings. As far as we know, Calamia et al. (2013) is the first work on this subject. However, the authors only consider the last spread value to represent the daily liquidity of an ETF. In many studies on ETFs, they have intuitively noted that an ETF is more liquid if its underlyings are expected to be more liquid. This may be easily understood given the fact that investors are more attracted by ETFs only when they are interested in buying their underlyings. From a macro point of view, we can then accept that the liquidity of an ETF is related to the liquidity of the underlying securities. But it is not evident that there is a relationship if we consider an intraday analysis. In this paragraph, we investigate this relationship by considering the bid-ask spread, as wall as the liquidity spread.

\subsubsection{Bid-ask spread}

The bid-ask spread of an ETF at time $t$ is computed by:

$$
S_{t}(0)=\frac{P_{t}^{\mathrm{BID}, 1}-P_{t}^{\mathrm{ASK}, 1}}{P_{t}^{\mathrm{MID}}}
$$

It corresponds to the liquidity spread $S_{t}(N)$ when the notional $N$ is equal to zero. In the same way, we consider $S_{t}^{i}(0)$ as the bid-ask spread of stock $i$. We can then define the bid-ask spread of the index as follows:

$$
S_{t}^{\text {Index }}(0)=\sum_{i \in \text { Index }} w_{i} S_{t}^{i}(0)
$$

where $w_{i}$ is the weight of stock $i$ in the index. To compute the bid-ask spread for a given period $\left[t_{\text {begin }}, t_{\text {end }}\right]$, we consider the time aggregation rule (4) and we weight the intraday spreads by the duration between two ticks. 
Following Broman and Shum (2013), the liquidity ratio $\mathcal{L} \mathcal{R}$ is defined as the ratio between the index spread and the ETF spread:

$$
\mathcal{L} \mathcal{R}_{t}=\frac{S_{t}^{\text {Index }}(0)}{S_{t}^{\text {ETF }}(0)}
$$

A ratio greater than one $\left(\mathcal{L R}_{t}>1\right)$ implies an improvement in liquidity, whereas a ratio smaller than one corresponds to a worsening of liquidity. In Figure 17, we report the boxplot of the liquidity ratio for four ETFs which track the EURO STOXX 50 index. We observe that the liquidity does not improve if we consider the ETF instead of the index.

Figure 17: Boxplot of the intraday liquidity ratio $\mathcal{L R}_{t}$ (EURO STOXX 50)

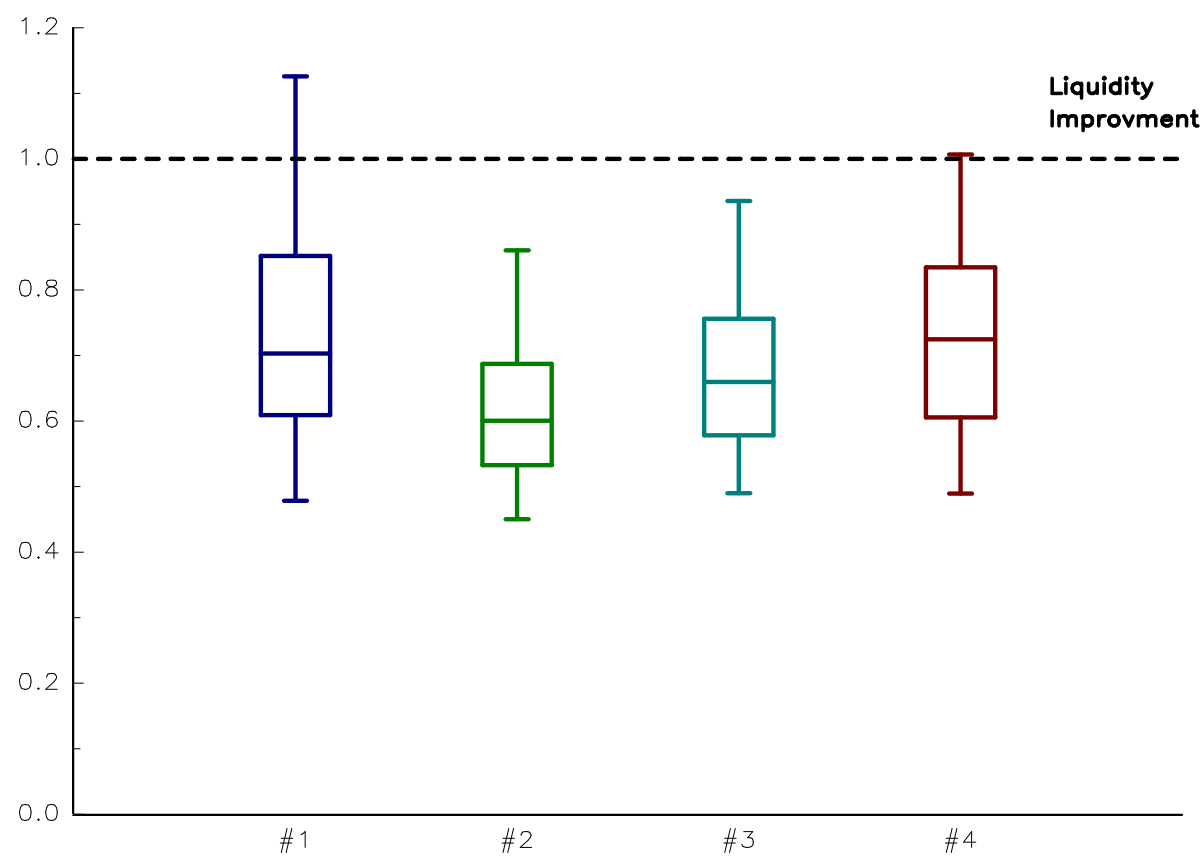

\subsubsection{Liquidity spread}

We define the liquidity spread of the index as follows:

$$
S_{t}^{\text {Index }}(N)=\sum_{i \in \text { Index }} w_{i} S_{t}^{i}\left(w_{i} \cdot N\right)
$$

where $w_{i}$ is the weight of stock $i$ in the index and $S_{t}^{i}\left(N_{i}\right)$ is the liquidity spread of stock $i$ for a given notional $N_{i}$. We notice that the liquidity spread of the index is more complicated to compute than the bid-ask spread of the index. Indeed, for each stock $i$, we have to compute the corresponding notional $N_{i}=w_{i} \cdot N$ to be traded and the liquidity spread $S_{t}^{i}\left(N_{i}\right)$ that corresponds to this notional.

Example 1 We consider the following liquidity spread (expressed in bps) for two stocks $A$ 
and $B$ :

\begin{tabular}{|r|cc|}
\hline Notional & Stock $A$ & Stock $B$ \\
\hline $0 K €$ & 6 & 8 \\
$300 K €$ & 8 & 9 \\
$500 K €$ & 10 & 10 \\
$700 K €$ & 12 & 11 \\
$1 M €$ & 14 & 12 \\
\hline
\end{tabular}

Suppose that the composition of the index is $70 \%$ of stock $A$ and $30 \%$ of stock B. The bid-ask spread of the index is equal to:

$$
S_{t}^{\text {Index }}(0)=0.70 \cdot 6+0.30 \cdot 8=6.6 \mathrm{bps}
$$

If we now want to trade a notional of $1 M €$ for the index, it means that we have to $0.7 \mathrm{M} €$ for stock $A$ and $0.3 M €$ for stock $B$. In this case, we have:

$$
S_{t}^{\text {Index }}(1 M €)=0.70 \cdot 12+0.30 \cdot 9=11.1 \mathrm{bps}
$$

Figure 18: Boxplot of the intraday liquidity ratio $\mathcal{L R}_{t}(N)$ (EURO STOXX 50)

$\# 1$

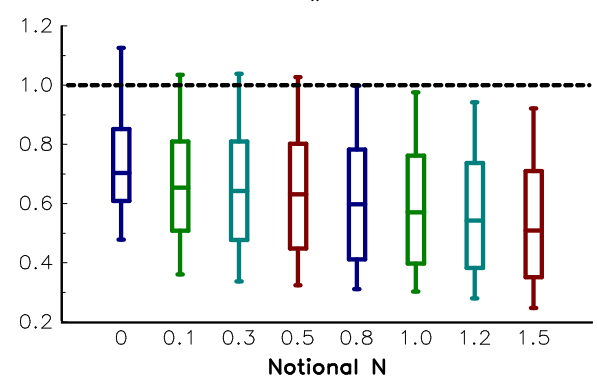

\#3

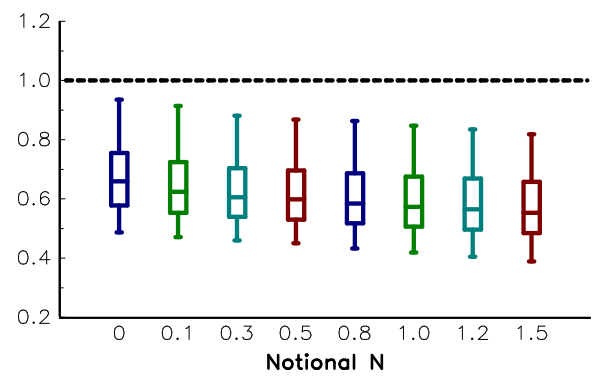

\#2

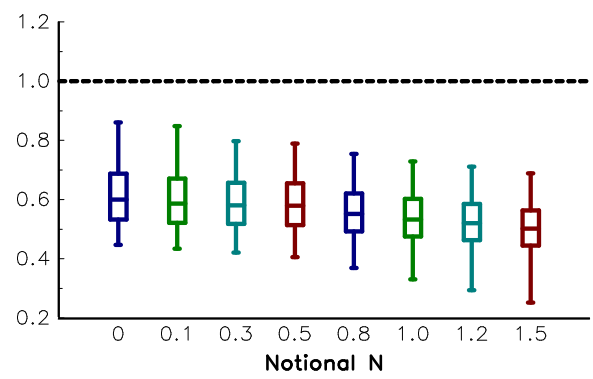

\#4

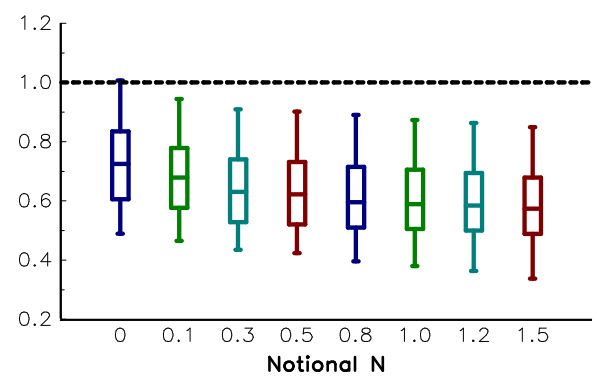

We define the liquidity ratio $\mathcal{L R}_{t}(N)$ in the same way as previously. However, there is a difference, because the index and ETF spreads now depend on the notional $N$. In Figure 17 , we report the boxplot of $\mathcal{L R}_{t}(N)$ for different values of $N$. We note that the liquidity ratio decreases with the notional.

Calamia et al. (2013) show that the first explanatory variable of the ETF liquidity is the spread of the underlying index. Using intraday data, we confirm that there may be a relationship between the daily liquidity spread of the ETF and the daily liquidity spread of the index. For instance, we report the scatterplot of these liquidity spreads in the case of 
the EURO STOXX 50 index for a notional equal to $1 \mathrm{M} €$ in Figure 19. We also indicate the fitted line obtained using linear regression. We verify that the slope of the curve is positive. However, the coefficient of determination $R^{2}$ is very low and is equal to $6 \%$ for ETFs \#1 and \#2 (b). For ETFs that present lower liquidity spreads, the relationship is stronger the $R^{2}$ is equal to $40 \%$ and $29 \%$ for ETFs \#3 (a) and \#4. One issue concerns the behaviour of this relationship on an intraday basis. In Figure 20, we report the scatterplot between the intraday spreads ${ }^{18} S_{t}^{\mathrm{ETF}}(N)$ and $S_{t}^{\operatorname{Index}}(N)$ for $N=1 \mathrm{M} €$. Curiously, we observe that the intraday liquidity spread of the ETF is not related to the intraday liquidity spread of the index.

Figure 19: Scatterplot of the daily spread $S_{t}^{\operatorname{Index}}(N)$ vs $S_{t}^{\text {ETF }}(N)$ (EURO STOXX 50)
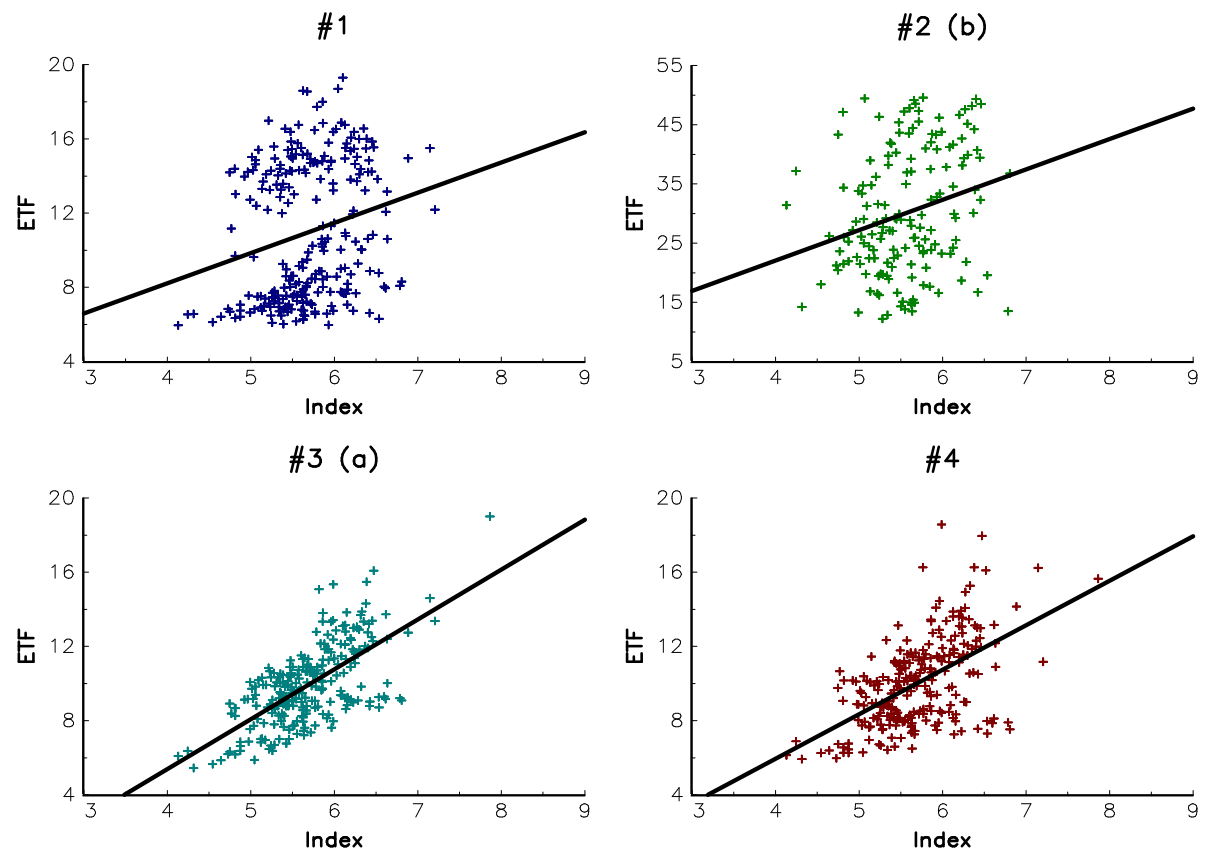

\section{Conclusion}

In this paper, we investigate the liquidity of European ETFs, both on a daily and intraday basis. The main result is that liquidity varies considerably between ETF providers. The fragmentation of the ETF market, the cross-listing of ETFs and the low transparency of the European ETF market are the most important factors that explain this situation.

Improving the efficiency of ETFs is the goal of supervision and regulation. As noted by Hassine and Roncalli (2013), the efficiency of ETFs depends on three main criteria: tracking difference, tracking error and liquidity. Some steps have been taken by regulators to define and highlight the first two criteria. Liquidity remains the factor forgotten by the supervisory authorities. However, it is undoubtedly a key issue in Europe if this market is to be improved, a situation that would help investors in their investment choices.

\footnotetext{
${ }^{18}$ As previously, the time period used to compute the liquidity spread is 30 minutes.
} 
Figure 20: Scatterplot between the intraday spreads (EURO STOXX 50)
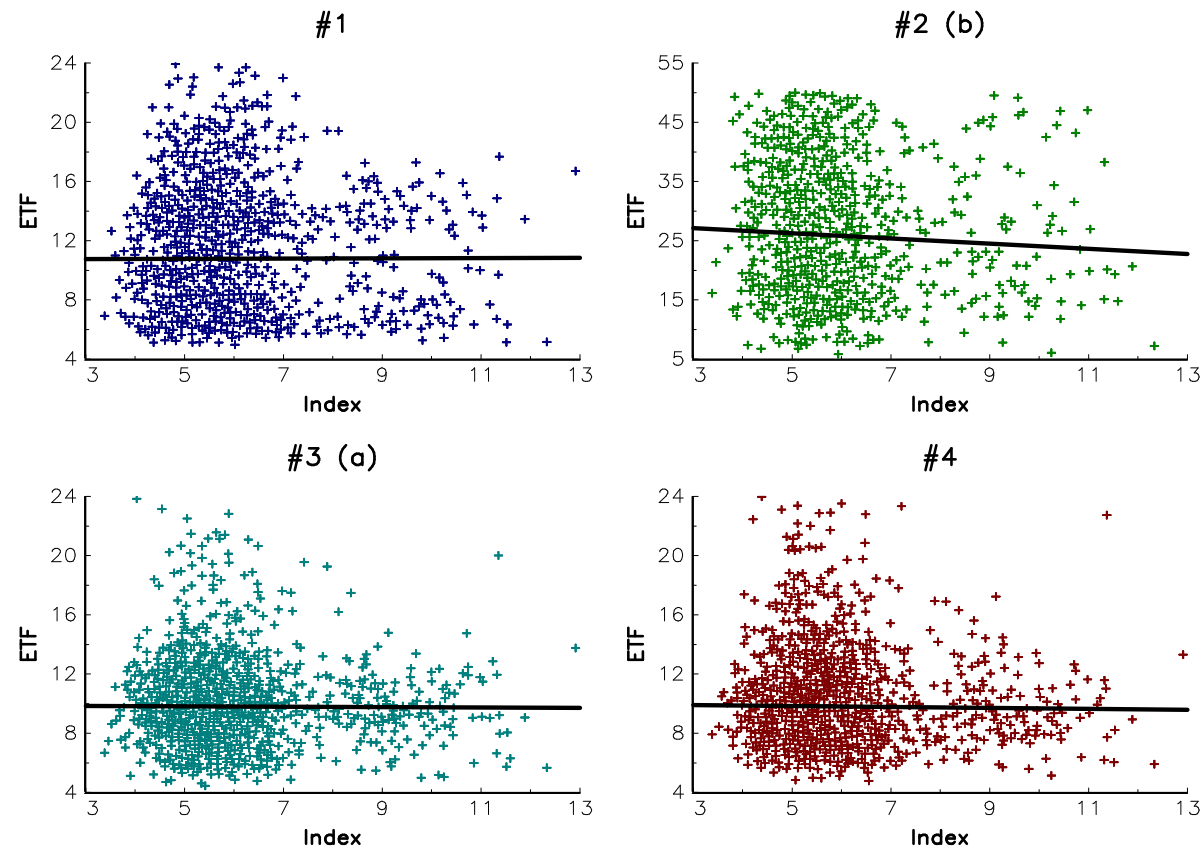

\section{References}

[1] Acerbi C. and Szekeres Z. (2013), Introduction to LiquidityMetrics: A Flexible Suite of Liquidity Measures, MSCI Research Insight.

[2] Amenc N., Goltz F., Gonzalez N., Shah N., Shirbini E. and Tessaromatis N. (2013), The EDHEC European ETF Survey 2012, EDHEC-Risk Institute.

[3] Aminud Y. (2002), Illiquidity and Stock Returns: Cross-Section and Time-Series Effects, Journal of Financial Markets, 5(1), pp. 31-56.

[4] Aminud Y. and Mendelson H. (1980), Dealership Market: Market-Making with Inventory, Journal of Financial Economics, 8(1), pp. 31-53.

[5] Aminud Y. and Mendelson H. (1986), Asset Pricing and The Bid-Ask Spread, Journal of Financial Economics, 17(2), pp. 223-249.

[6] Aminud Y. and Mendelson H. (1989), The Effects of Beta, Bid-Ask Spread, Residual Risk, and Size on Stock Returns, Journal of Finance, 44(2), pp. 479-486.

[7] Aminud Y. and Mendelson H. (1991), Liquidity, Asset Prices and Financial Policy, Financial Analysts Journal, 47(6), pp. 56-66.

[8] Barclay M. J., Christie W. J., Harris J. H., Kandel E. and Schultz P. H. (1999), The Effects of Market Reform on the Trading Costs and Depths of Nasdaq Stocks, Journal of Finance, pp. 1-34.

[9] Ben-Rephael A., Kadan O. and Wohl A. (2013), The Diminishing Liquidity Premium, Journal of Financial and Quantitative Analysis, forthcoming. 
Measuring the Liquidity of ETFs: An Application to the European Market

[10] BlackRock (2012), ETP Landscape: Global Handbook 2012, BlackRock Investment Institute.

[11] Brennan M.J. and Subrahmanyam A. (1996), Market Microstructure and Asset Pricing: On the Compensation for Illiquidity in Stock Returns, Journal of Financial Economics, 41(3), pp. 441-464.

[12] Broman M. and Shum P. (2013), Liquidity Improvement, Exchange Traded Funds Flows and Mispricing, SSRN, www.ssrn.com/abstract=2361514.

[13] Calamia A., Deville L. and Riva F. (2013), Liquidity in European Equity ETFs: What Really Matters?, Bankers Markets 83 Investors, 124, pp. 60-73.

[14] Degryse H., De Jong F. and Van Kervel V. (2011), The Impact of Dark Trading and Visible Fragmentation on Market Quality, CEPR Discussion Paper, 8630.

[15] Deville L., Gresse C. and De Séverac B. (2012), Direct and Indirect Effects of Index ETFs on Spot-Futures Pricing and Liquidity: Evidence from the CAC 40 Index, European Financial Management.

[16] De Winne R., Platten I. and Gresse C. (2013), Liquidity and Risk Sharing Benefits from the Introduction of an ETF, SSRN, www.ssrn. com/abstract=1253181.

[17] Easley D. and O'Hara M. (1987), Price, Trade Size, and Information in Securities Markets, Journal of Financial Economics, 19(1), pp. 69-90.

[18] Easley D. and O'Hara M. (1991), Order Form and Information in Securities Markets, Journal of Finance, 46(3), pp. 905-927.

[19] Eleswarapu R. (1997), Cost of Transacting and Expected Returns in the Nasdaq Market, Journal of Finance, 52(5), pp. 2113-2127.

[20] ETFGI (2014), Global ETF and ETP Industry Insights: Year End 2013.

[21] Giulianini P. (2012), Understanding ETF Trading and Liquidity in Europe, in B.R. Bruce (Ed.), Guide to ETFs $\& 3$ Indexing in European Markets, Institutional Investor Journals.

[22] Glosten L.R. and Milgrom P.R. (1985), Bid, Ask and Transaction Prices in a Specialist Market with Heterogeneously Informed Traders, Journal of Financial Economics, 14(1), pp. 71-100.

[23] Hassine M. and Roncaldi T. (2013), Measuring Performance of Exchange Traded Funds, Journal of Index Investing, 4(3), pp. 57-85.

[24] Keim D.B. and Madhavan A. (1996), The Upstairs Market for Large-block Transactions: Analysis and Measurement of Price Effects, Review of Financial Studies, 9(1), pp. 1-36.

[25] Parlour C.A. and Seppi D.J. (2008), Limit Order Markets: A Survey, Handbook of Financial Intermediation and Banking, 5, pp. 63-95.

[26] Sarr A. and Lybek T. (2002), Measuring Liquidity in Financial Markets, IMF Working Papers, 02/232. 

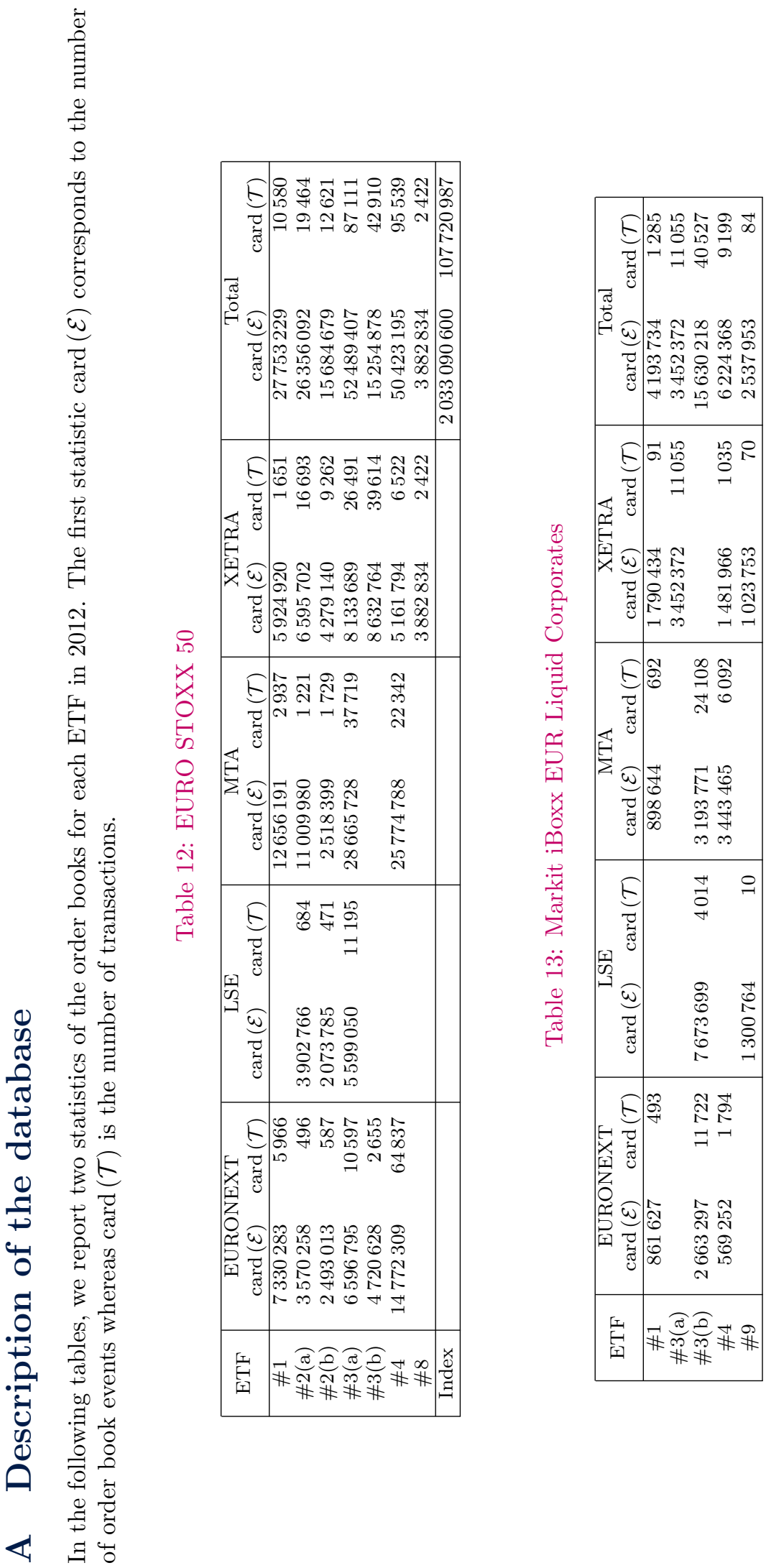

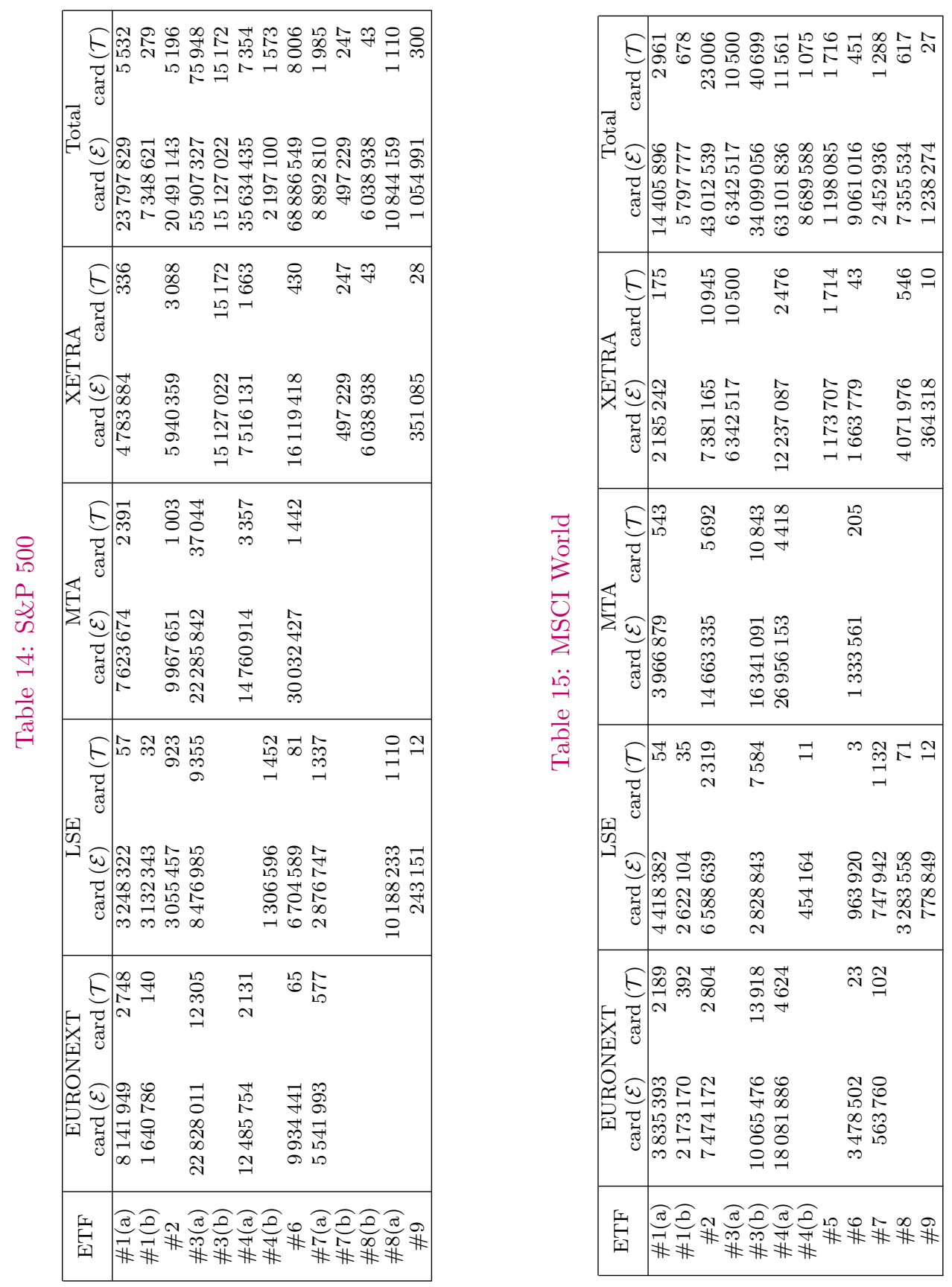


\section{B Example of a liquidity spread calculation}

Let us illustrate the spread calculation with the order book of the Lyxor EURO STOXX 50 ETF recorded in NYSE Euronext Paris given in Table 16. The traditional spread is 5.696 bps whereas the mid price is 26.333 . Suppose that we would like to trade $1 \mathrm{M} €$. In this case, it is equivalent to trade 37976 shares. The results are given in Table 17. We notice that the first limit is enough to absorb this trade. This explains that the spread for a notional of $1 \mathrm{M} €$ is equal to the traditional spread. If we consider a notional of $5 \mathrm{M} €$, we have to go to the three limits. In this case, the spread is higher and is equal to $8.570 \mathrm{bps}$. If the notional is $10 \mathrm{M} €$, the number of shares to trade is 379759 . The cumulative quantity of the five limits is then insufficient, given that the coefficient $S_{t_{j}}(N)$ is larger than one.

Table 16: Limit order book

\begin{tabular}{|c|rc:rc|}
\hline$i$ & \multicolumn{2}{|c|}{ Buy orders } & \multicolumn{2}{c|}{ Sell orders } \\
& \multicolumn{1}{|c|}{$Q_{t_{j}}^{\mathrm{BID}, \mathrm{i}}$} & $P_{t_{j}}^{\mathrm{BID}, \mathrm{i}}$ & $Q_{t_{j}}^{\mathrm{ASK}, \mathrm{i}}$ & $P_{t_{j}}^{\mathrm{ASK}, \mathrm{i}}$ \\
\hline 1 & 65201 & 26.325 & 70201 & 26.340 \\
2 & 85201 & 26.320 & 116201 & 26.345 \\
3 & 105201 & 26.315 & 107365 & 26.350 \\
4 & 76500 & 26.310 & 35000 & 26.355 \\
5 & 20000 & 26.305 & 35178 & 26.360 \\
\hline
\end{tabular}

Table 17: Computing the spread for a given notional $N$

\begin{tabular}{|c|c|c|c|c|c|c|}
\hline \multirow[t]{2}{*}{$k$} & \multicolumn{2}{|c|}{$N=1 \mathrm{M} €$} & \multicolumn{2}{|c|}{$N=5 \mathrm{M} €$} & \multicolumn{2}{|c|}{$N=10 \mathrm{M} €$} \\
\hline & $\bar{Q}_{t_{j}}^{\mathrm{BID}, \mathrm{i}}$ & $\bar{Q}_{t_{j}}^{\mathrm{ASK}, \mathrm{i}}$ & $\bar{Q}_{t_{j}}^{\mathrm{BID}, \mathrm{i}}$ & $\bar{Q}_{t_{j}}^{\mathrm{ASK}, \mathrm{i}}$ & $\bar{Q}_{t_{j}}^{\mathrm{BID}, \mathrm{i}}$ & $\bar{Q}_{t_{j}}^{\mathrm{ASK}, \mathrm{i}}$ \\
\hline 1 & 37976 & 37976 & 65201 & 70201 & 65201 & 70201 \\
\hline 2 & 0 & 0 & 85201 & 116201 & 85201 & 116201 \\
\hline 3 & 0 & 0 & 39478 & 3478 & 105201 & 107365 \\
\hline 4 & 0 & 0 & 0 & 0 & 76500 & 35000 \\
\hline 5 & 0 & 0 & 0 & 0 & 20000 & 35178 \\
\hline $\bar{\sum}_{i=1}^{5^{-}-} \bar{Q}_{t_{j}, \bar{i}}^{\overline{\boldsymbol{e}}}$ & $\overline{37} \overline{97} \overline{6}$ & $\overline{379} \overline{7} \overline{6}$ & $\overline{189} \overline{880}$ & $\overline{18} \overline{98} \overline{0}$ & $\overline{352} \overline{103}$ & $\overline{36} \overline{3} \overline{94} \overline{5}$ \\
\hline $\bar{P}_{t_{j}, i}^{\bullet}$ & 26.325 & 26.340 & 26.321 & 26.343 & 26.316 & 26.348 \\
\hline$c_{t_{j}}(N)$ & & 00 & & & & \\
\hline$S_{t_{j}}(N)$ & & & & & & \\
\hline
\end{tabular}

\section{Additional figures}


Figure 21: Average intraday trading volume (S\&P 500)
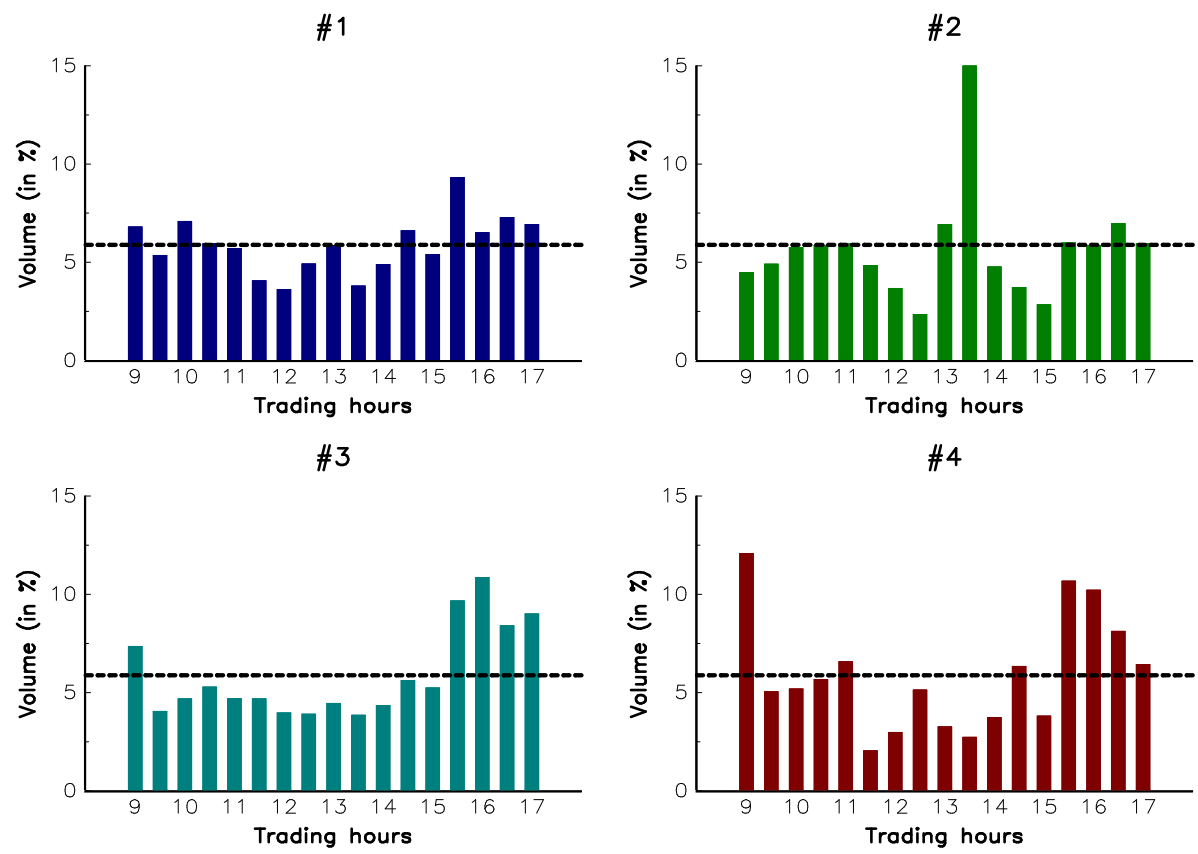

Figure 22: Average intraday trading volume (MSCI WORLD)
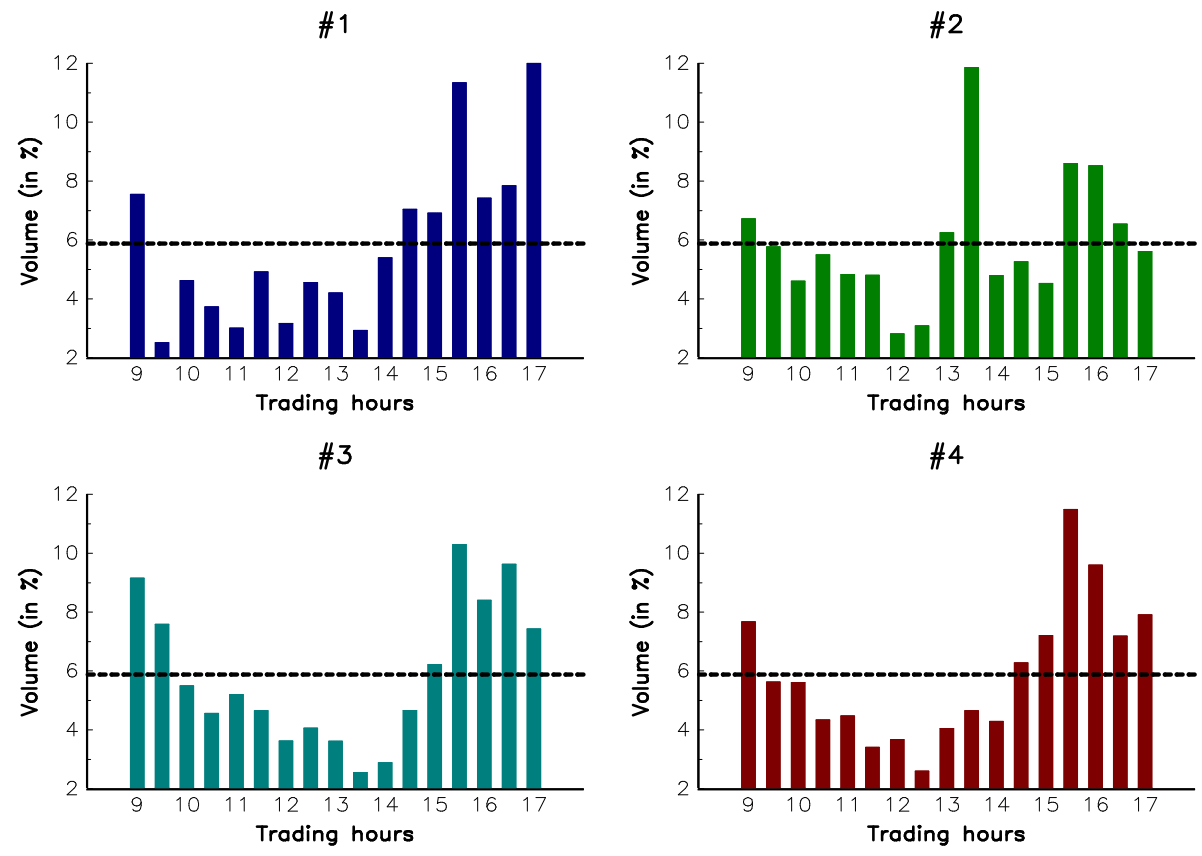
Figure 23: Average intraday trading volume (IBOXX EUR LIQUID)
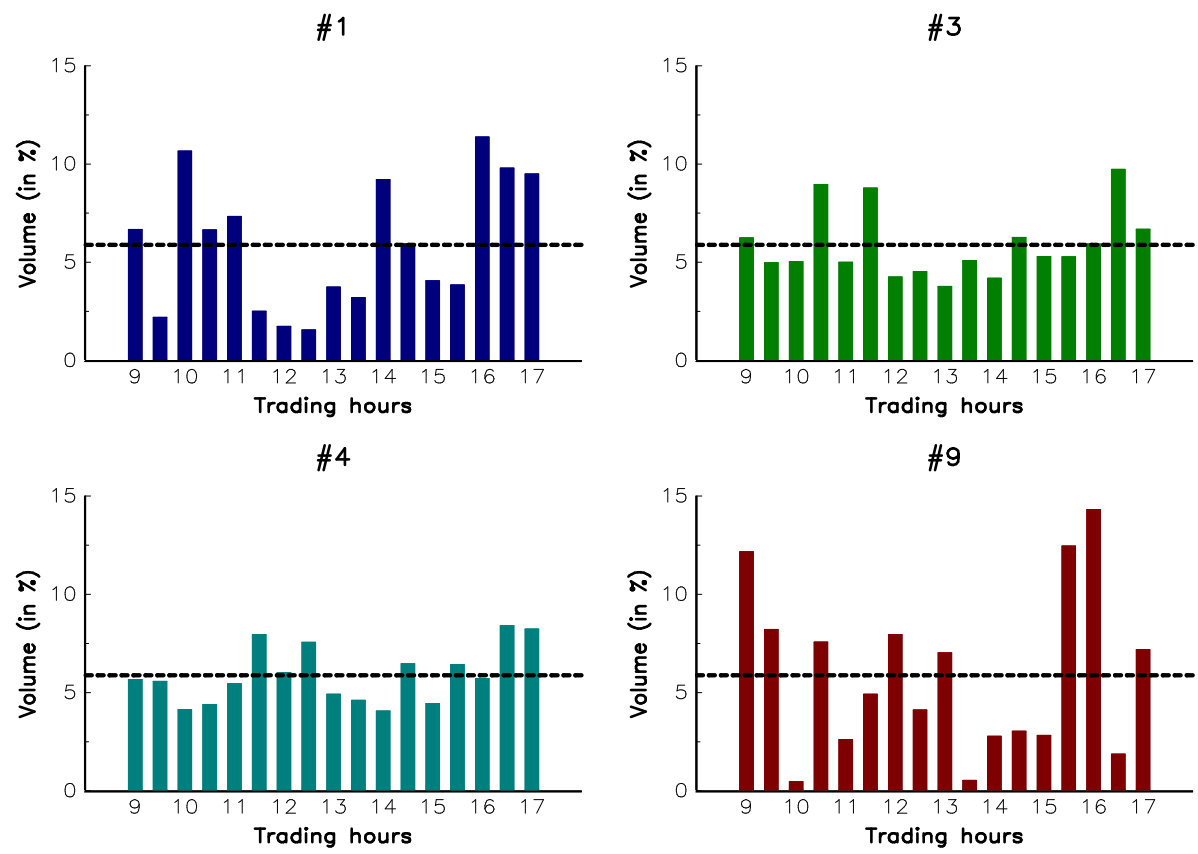

Figure 24: Boxplot of the intrady best limit volume $Q^{\mathrm{MEAN,1}}$ (EURO STOXX 50)
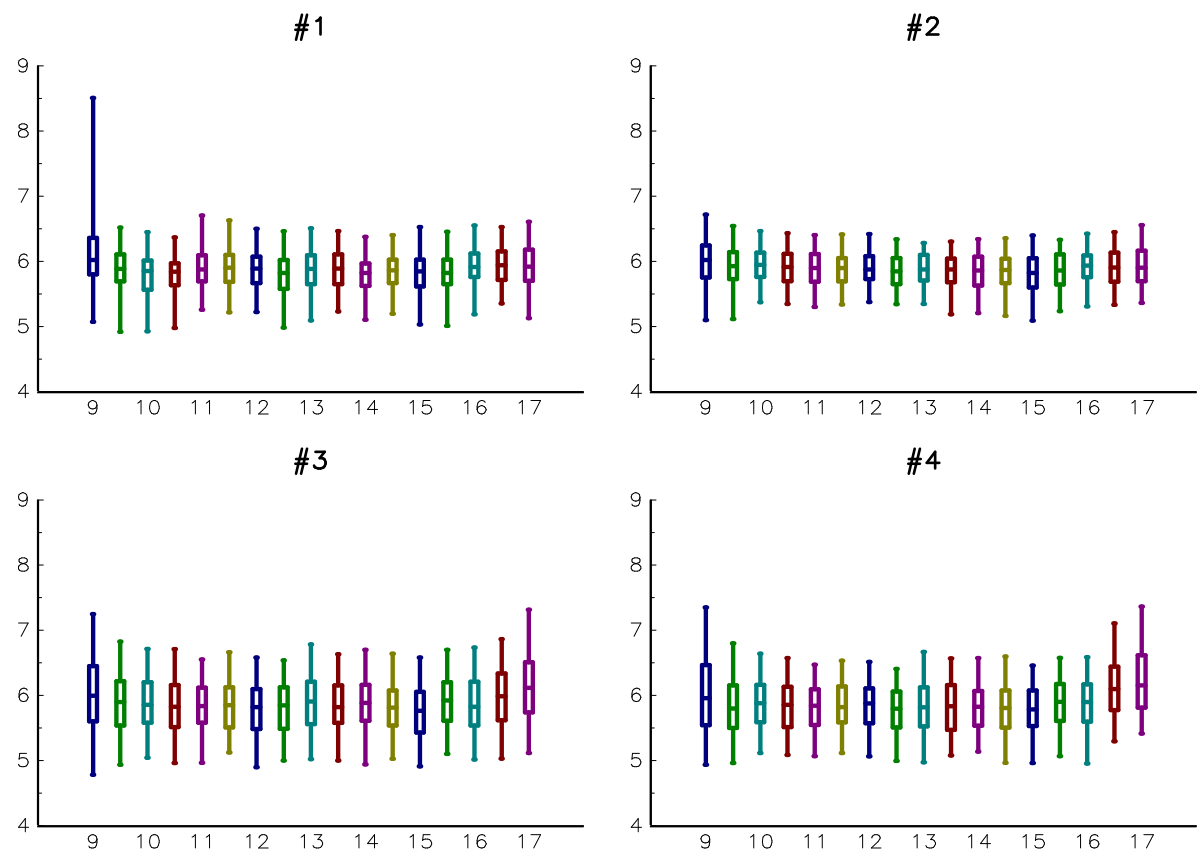
Figure 25: Boxplot of the intraday spread (EURO STOXX 50)
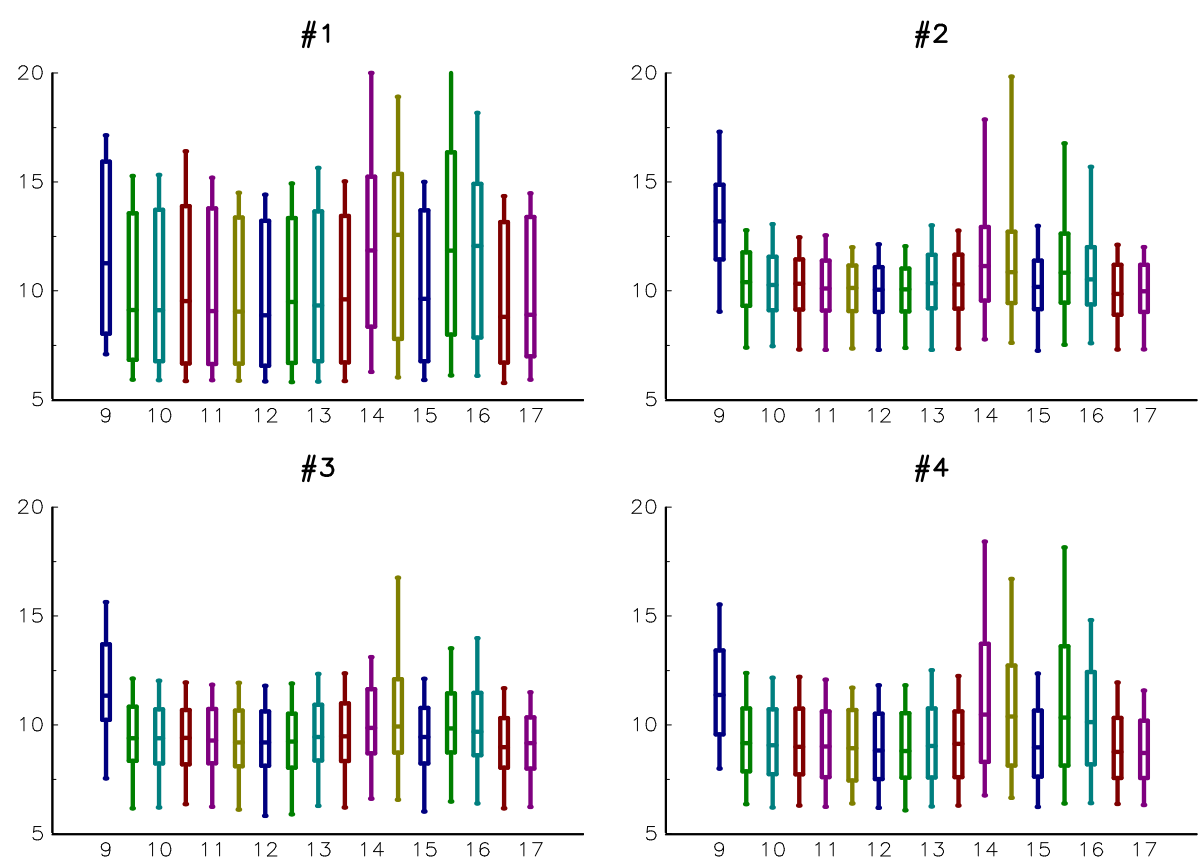

Figure 26: Boxplot of the intraday spread (MSCI WORLD)
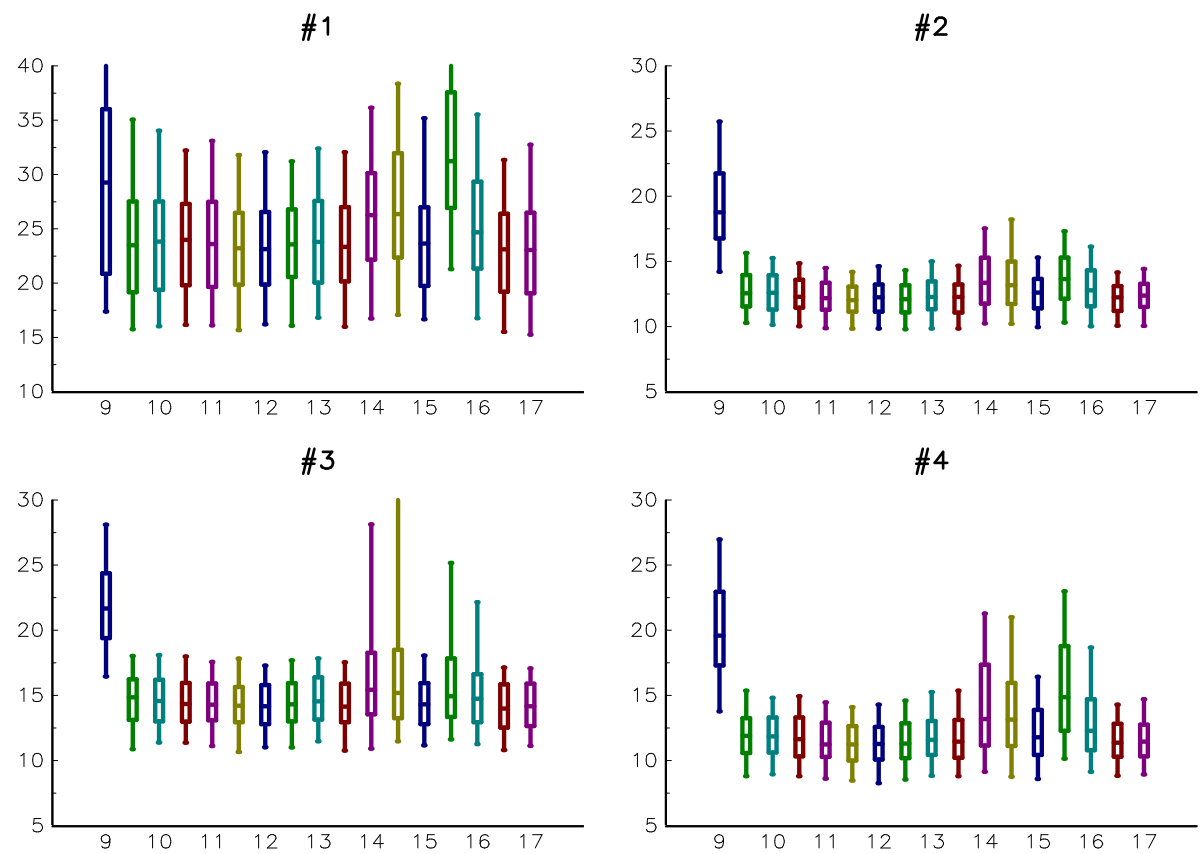
Figure 27: Boxplot of the intraday spread (IBOXX EUR LIQUID)
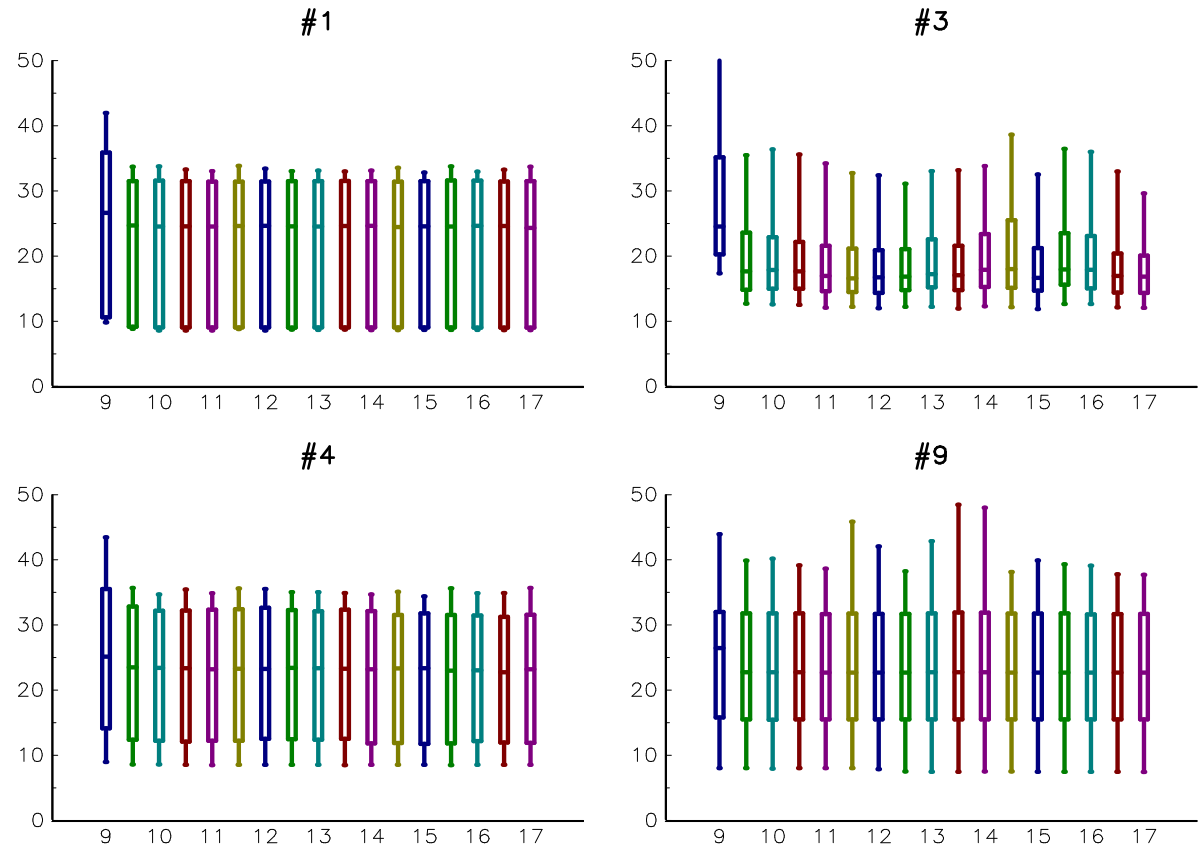

Figure 28: Boxplot of the intraday liquidity notional (EURO STOXX 50)
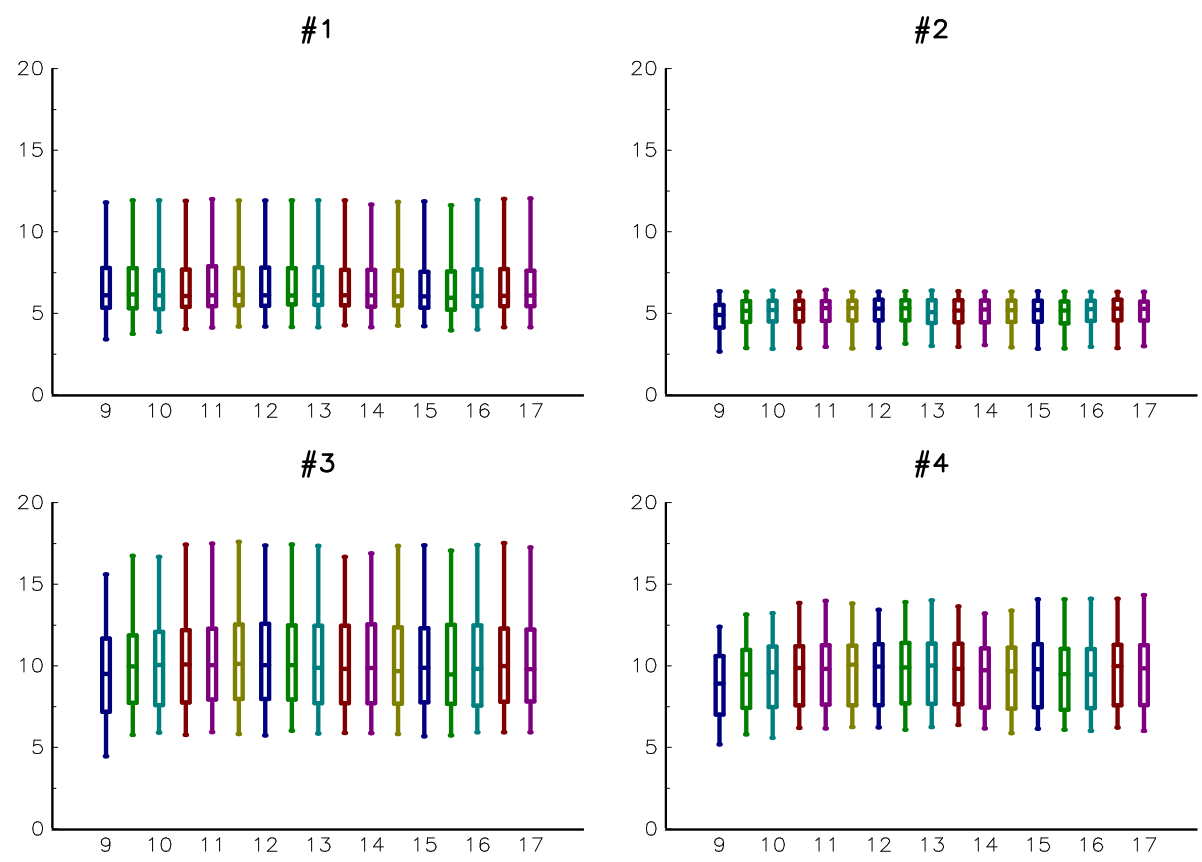